ESAIM: M2AN 48 (2014) 411-431

DOI: $10.1051 / \mathrm{m} 2 \mathrm{an} / 2013113$
ESAIM: Mathematical Modelling and Numerical Analysis

www.esaim-m2an.org

\title{
ASYMPTOTICS OF A TIME-SPLITTING SCHEME FOR THE RANDOM SCHRÖDINGER EQUATION WITH LONG-RANGE CORRELATIONS
}

\author{
Christophe Gomez ${ }^{1}$ And Olivier Pinaud ${ }^{2}$
}

\begin{abstract}
This work is concerned with the asymptotic analysis of a time-splitting scheme for the Schrödinger equation with a random potential having weak amplitude, fast oscillations in time and space, and long-range correlations. Such a problem arises for instance in the simulation of waves propagating in random media in the paraxial approximation. The high-frequency limit of the Schrödinger equation leads to different regimes depending on the distance of propagation, the oscillation pattern of the initial condition, and the statistical properties of the random medium. We show that the splitting scheme captures these regimes in a statistical sense for a time stepsize independent of the frequency.

Résumé. Nous nous intéressons au comportement asymptotique d'un schéma de time-splitting pour l'équation de Schrödinger avec potential aléatoire de faible amplitude, oscillant rapidement en temps et en espace, et présentant des corrélations longue portée. Cette équation décrit par exemple la propagation d'une onde dans un milieu aléatoire dans le cadre de l'approximation paraxiale. La limite haute-fréquence de l'équation de Schrödinger mène à différents régimes selon la distance de propagation, la fréquence d'oscillation de la condition initiale, et la statistique du milieu aléatoire. Nous montrons que le schéma de splitting capture statistiquement ces régimes asymptotiques pour un pas de discrétisation en temps indépendant de la fréquence d'oscillation.
\end{abstract}

Mathematics Subject Classification. 65M12, 65M70, 65C30, 60H15.

Received September 3, 2013.

Published online February 20, 2014.

\section{INTRODUCTION}

Time-splitting schemes for the Schrödinger equation have been widely used for applications that range from quantum transport to wave propagation in random media $[3,4,11,13,14]$. In this work, we are interested in the asymptotic (high frequency, or semi-classical) behavior of solutions to a weakly random Schrödinger equation and how the asymptotic regimes are captured by the splitting scheme. It is a natural question to wonder how large can be chosen the stepsizes so as to lower the computational cost while recovering the correct limiting regimes. We consider random media with long-range correlations (also referred to as slowly decaying correlations, see Sect. 2 for a mathematical definition of such a property), as opposed to short-range correlations.

\footnotetext{
Keywords and phrases. Random Schrödinger equation, long-range correlations, high frequency asymptotics, splitting scheme.

1 Laboratoire d'Analyse, Topologie, Probabilités, UMR 7353, Aix-Marseille Université, Marseille, France.

christophe.gomez@latp.univ-mrs.fr

2 Department of Mathematics, Colorado State University, Fort Collins, CO, USA. pinaud@math.colostate.edu
} 
Such media are of interest for instance when waves propagate through a turbulent atmosphere or the earth upper crust $[6,16]$. As will be described below, long-range correlations lead to a richer asymptotical structure than short-range correlations. In wave propagation problems, the Schrödinger equation is seen as the paraxial or parabolic approximation of the wave equation and describes the evolution of the envelope function [18]. The randomness models for instance fluctuations in the sound speed profile of the propagation medium, see [7] for more examples. We consider a free Schrödinger equation perturbed by a random potential $V$, with dependence on both time and space, and which has a weak amplitude $\varepsilon^{(1-\gamma) / 2} \ll 1$ : for $d \geq 1$, let $\phi$ be the solution to

$$
\begin{gathered}
\mathrm{i} \frac{\partial \phi}{\partial t}+\frac{1}{2} \Delta \phi-\varepsilon^{(1-\gamma) / 2} V\left(\frac{t}{\varepsilon^{\gamma}}, x\right) \phi=0, \quad x \in \mathbb{R}^{d}, \quad t>0 \\
\phi(0, x)=\phi_{0}^{\varepsilon}(x),
\end{gathered}
$$

where $\phi_{0}^{\varepsilon} \in \mathcal{C}_{c}^{\infty}\left(\mathbb{R}^{d}\right)$ may depend on $\varepsilon, \gamma \in[0,1)$ and all physical constants have been set to one. The parameter $\gamma$ is here to account for some possible different scales of oscillations of the potential between time and space. In the wave propagation context, the time variable $t$ is replaced by the variable of the propagation axis. Let us remark that because of the small amplitude of the random fluctuations, one needs to rescale the time and space variables in order to observe a non-trivial behavior of the solutions:

$$
\phi^{\varepsilon}(t, x)=\phi\left(\frac{t}{\varepsilon^{s}}, \frac{x}{\varepsilon^{s}}\right),
$$

where $s \in(0,1]$ is called throughout the paper the propagation scale parameter. When $t$ and $x$ are both of order one (i.e. when $s=\gamma=0$ ) with respect to $\varepsilon$, asymptotic expressions of $\phi$ are simply given by perturbation theory and the stochastic effects induced by the random fluctuations remain small. Here, we are interested in regimes in which stochastic effects cumulate during the propagation and become of order one.

The asymptotic behavior of (1.1) as $\varepsilon \rightarrow 0$ is now well-established. It has been shown in several references $[1,2,8,9]$ that various regimes take place at different scales of space and time, and that these scales are intimately related to the statistical properties of the random potential $V$. A main distinction is whether the potential has short-range correlations or not. In the short-range case, the wavefunction and the wave energy evolve at the same scale (time and space) of order $\varepsilon^{-1}(s=1$ in (1.2)) [1]. The situation is different in the long-range case where wavefunction and energy are affected by the random medium at different scales. Various asymptotic regimes of propagation can be exhibited depending on the propagation scale parameter $s$, the oscillation pattern of the initial condition, and the correlation structure of the potential. We are mainly interested in the following three regimes described below:

(i) The phase modulation regime, taking place for relatively short distances of propagation. It corresponds to the scaling $s=1 / 2 \kappa_{\gamma}$, where $\kappa_{\gamma}>\frac{1}{2}$ is a parameter to be defined later on and depending on the statistical properties of the potential $V$. In this regime, the law of the asymptotic wavefunction is roughly the law of the free wavefunction modulated by a fractional Brownian motion with Hurst index $\kappa_{0}$ [2], see (2.7).

(ii) The wave decoherence regime, taking place for moderate distances of propagation. It arises for values of $s \in\left(1 / 2 \kappa_{\gamma}, 1\right)$ that depend on both the initial condition and the potential. This regime does not exist in the short-range case, and the initial condition needs to exhibit oscillations at a lower frequency than the medium (which is $\varepsilon^{-s}$ in rescaled variables) for the regime to be observed [9].

(iii) The radiative transfer regime, taking place for large distances of propagation. The corresponding scaling is $s=1$. In the long-range case, the collision operator of the obtained transport equation is singular, while it is smooth in the short-range case [8]. The expression of the collision operator depends on correlation function of the random medium and on the parameter $\gamma$. Transport equations generally describe quadratic quantities in the wavefunction, see [1] for a review of derivation of transport equations for high frequency random Schrödinger equations. 
In this work, we will only consider the long-range correlations case and postpone the short-range correlation case as well as numerical simulations to a future work. With the rescaled wavefunction $\phi^{\varepsilon}$ defined by (1.2), the Schrödinger equation (1.1) becomes

$$
\begin{gathered}
\mathrm{i} \varepsilon^{s} \frac{\partial \phi^{\varepsilon}}{\partial t}+\frac{\varepsilon^{2 s}}{2} \Delta \phi^{\varepsilon}-\varepsilon^{(1-\gamma) / 2} V\left(\frac{t}{\varepsilon^{s+\gamma}}, \frac{x}{\varepsilon^{s}}\right) \phi^{\varepsilon}=0, \quad x \in \mathbb{R}^{d}, \quad t>0 \\
\phi^{\varepsilon}(0, x)=\phi_{0}^{\varepsilon}\left(\frac{x}{\varepsilon^{s}}\right) .
\end{gathered}
$$

In order to define the splitting scheme, consider a fixed stepsize $h>0, n \in \mathbb{N}^{*}$ and let

$$
\mathcal{A}_{\varepsilon}=\frac{\mathrm{i} \varepsilon^{s}}{2} \Delta, \quad \text { and } \quad \mathcal{B}_{n, h}^{\varepsilon}(x)=\frac{1}{\varepsilon^{s-(1-\gamma) / 2}} \int_{(n-1) h}^{n h} V\left(\frac{u}{\varepsilon^{s+\gamma}}, \frac{x}{\varepsilon^{s}}\right) \mathrm{d} u
$$

The standard first-order semi-discrete split-step scheme then reads

$$
\Psi_{n}^{\varepsilon}=\mathrm{e}^{h \mathcal{A}_{\varepsilon}} \mathrm{e}^{-\mathrm{i} \mathcal{B}_{n, h}^{\varepsilon}} \Psi_{n-1}^{\varepsilon}, \quad \text { for } n=1,2, \ldots, \quad \text { with } \quad \Psi_{0}^{\varepsilon}(x)=\phi_{0}^{\varepsilon}\left(\frac{x}{\varepsilon^{s}}\right) .
$$

Let us note that the order in time of the scheme can be improved by taking other combinations of exponentials of operators, see e.g. $[15,17]$. We will not discretize the spatial variable $x$ here, the reason for that being that the semi-group $\mathrm{e}^{h \mathcal{A}_{\varepsilon}}$ can be computed at low cost with a high accuracy using spectral methods as Fast Fourier Transforms provided the spatial stepsize $\Delta x$ verifies $\Delta x \ll \varepsilon^{s}$. The computation does not introduce a constraint on the time stepsize $h$ since the time variable is treated exactly. Absorbing boundary conditions are needed since the wavefunction solves the Schrödinger in the whole space, and one can find in [10] boundary conditions compatible with FFT.

It is well-established that splitting techniques are efficient numerical methods for the resolution of semiclassical Schrödinger equations as the ones we are considering, see for instance [11,13]. It was shown in [3] for the short-range case in the transport scaling $s=1$ with $\gamma=0$, that the scheme captures the correct transport regime if the stepsize is like $\varepsilon h^{\prime}$, where $h^{\prime} \ll 1$ independently of $\varepsilon$. It is a statistical result, as the convergence holds in probability and not almost surely. A larger stepsize would still lead to a transport regime, but with a wrong collision cross-section. Notice that the constraint $h=\varepsilon h^{\prime}$ is an improvement of the consistency constraint for capturing the wavefunction trajectory: indeed, the local error estimate between the exact solution and its approximation after one single step is readily shown to be $([\cdot, \cdot]$ denotes commutator between two operators):

$$
\left\|\phi^{\varepsilon}(h)-\Psi_{1}^{\varepsilon}\right\|_{L^{2}} \leq \frac{h^{2}}{\varepsilon}\left\|[\varepsilon \Delta, \sqrt{\varepsilon} V] \mathrm{e}^{-\frac{i \varepsilon}{2} \Delta} \phi_{0}^{\varepsilon}\right\|_{L^{2}}+\frac{h^{2}}{\varepsilon}\left\|\phi_{0}^{\varepsilon}\right\|_{L^{2}}\|V\|_{L_{t, x}^{\infty}}^{2},
$$

so that if $\phi_{0}^{\varepsilon}$ and $V$ are smooth enough (e.g. $\varepsilon\left\|\nabla \phi_{0}^{\varepsilon}\right\|_{L^{2}}+\|\nabla V\|_{L_{t, x}^{\infty}}+\|\Delta V\|_{L_{t, x}^{\infty}} \leq C$ ), then the local error is controlled by $C h^{2} \varepsilon^{-3 / 2}$. The global error is $h \varepsilon^{-3 / 2}$ and one needs $h \ll \varepsilon^{3 / 2}$ for the scheme to be consistent, which is to be compared to $h \ll \varepsilon$ for capturing the transport regime in probability. The scheme is always stable since it preserves the $L^{2}$ norm:

$$
\left\|\Psi_{n}^{\varepsilon}\right\|_{L^{2}}=\left\|\Psi_{0}^{\varepsilon}\right\|_{L^{2}}, \quad \forall n \in \mathbb{N} .
$$

We are interested in this work in finding the constraints on the stepsize $h$ for the splitting scheme to capture the three asymptotic regimes described above. We will show that for most of our parameters configurations, the three regimes are captured for all $h>0$, independently of $\varepsilon$. Such a result is a consequence of several facts: we consider convergence in a statistical sense and not of the trajectories; and the random medium has long-range correlations and oscillates fast enough. The consequence is that statistically, the Laplacian and the potential evolve at different scales in regimes (i) and (ii) so that there is little interaction between them and their commutator is small in some sense. Then, it suffices to take the limit $\varepsilon \rightarrow 0$ in the potential term, which leads to the right behavior for all $h>0$. The situation is slightly different in the transport regime where decoupling 
occurs only when $\gamma>0$, that is when the potential oscillates faster in the time variable than in the spatial variables. When $\gamma=0$, Laplacian and potential interact to yield a different collision operator as in [1].

The organization of the paper is as follows. In Section 2, we present our main asumptions and our main results. Theorem 2.1 addresses regime (i), Theorems 2.2 and 2.3 regime (ii), and Theorem 2.3 regime (iii). The respective proofs are given in Sections 3-6. Our proofs will remain at the formal level. They can be made rigorous by using the techniques of $[2,3,8,9]$, which are beyond the scope of this paper.

\section{MAIn RESUlts}

In this section, we state our main results for regimes (i), (ii) and (iii). We present first some notation and our assumptions on the random potential.

The Fourier transform and inverse Fourier transform of a regular function $f$ are respectively given by

$$
\widehat{f}(k)=\int_{\mathbb{R}^{d}} \mathrm{~d} x \mathrm{e}^{-\mathrm{i} k \cdot x} f(x) \quad \text { and } \quad f(x)=\frac{1}{(2 \pi)^{d}} \int_{\mathbb{R}^{d}} \mathrm{~d} k \mathrm{e}^{\mathrm{i} x \cdot k} \widehat{f}(k) .
$$

Long-range correlations. We use the same setting as [2], see therein for the details. Namely, $V$ is mean-zero Gaussian stationary Markov process with correlation function

$$
\begin{aligned}
\mathbb{E}\{V(t+s, x+y) V(s, y)\} & =R(t, x)=\frac{1}{(2 \pi)^{d}} \int_{\mathbb{R}^{d}} \mathrm{~d} k \mathrm{e}^{\mathrm{i} k \cdot x} \tilde{R}(t, k) \\
& =\frac{1}{(2 \pi)^{d+1}} \int_{\mathbb{R}^{d+1}} \mathrm{~d} k \mathrm{~d} \omega \mathrm{e}^{\mathrm{i} k \cdot x} \mathrm{e}^{\mathrm{i} \omega t} \widehat{R}(\omega, k),
\end{aligned}
$$

where

$$
\tilde{R}(t, k)=\mathrm{e}^{-\mu|k|^{2 \beta}|t|} \frac{a(k)}{|k|^{2 \alpha+d-2}}, \quad \text { and } \quad \widehat{R}(\omega, k)=\frac{2 \mu|k|^{2 \beta} a(k)}{|k|^{2 \alpha+d-2}\left(\omega^{2}+\mu^{2}|k|^{4 \beta}\right)},
$$

for some $\mu>0$ and $a \in \mathcal{C}_{c}^{\infty}\left(\mathbb{R}^{d}\right)$ with $a(0)>0$. The parameters $\alpha$ and $\beta$ verify

$$
\alpha+\beta>1, \quad \frac{1}{2}<\alpha<1, \quad 0<\beta \leq \frac{1}{2} .
$$

The parameter $\alpha$ can be seen as a quantification of the correlation range in space and $\beta$ of the correlation between the space and time variables. Such potential has long-range correlations since $R(t, x)$ is not integrable [2].

Remark on the numerical computation of $\mathcal{B}_{n, h}^{\varepsilon}$. We address here the question as to how the coefficients $\mathcal{B}_{n, h}^{\varepsilon}$ can be computed numerically with efficiency. It the potential $V$ is provided by the problem, then the integral in time in $\mathcal{B}_{n, h}^{\varepsilon}$ can be discretized using a quadrature rule independent of $\varepsilon$, despite the fast oscillations. This is again a consequence of the fact that we are interested in the statistical properties of the wavefunction. To see it, it suffices to replace $\mathcal{B}_{n, h}^{\varepsilon}$ by a discrete version in the different proofs and to observe that there are no $\varepsilon$-dependent constraints of the quadrature stepsize in order to recover the correct asymptotics. In practice, one needs of course to average over a large enough number of realizations for this to hold. If the power spectrum $\widehat{R}(\omega, k)$ is provided, the coefficient $\mathcal{B}_{n, h}^{\varepsilon}$ can be directly generated once and for all by the following formula that can be discretized using FFT in $\omega$ and $p$ :

$$
\mathcal{B}_{n, h}^{\varepsilon}(x)=\frac{\varepsilon^{(1-s) / 2}}{(2 \pi)^{d+1}} \int_{\mathbb{R}^{d+1}} \mathrm{~d} G(\mathrm{~d} \omega, \mathrm{d} p) \mathrm{e}^{\mathrm{i} \omega n h} \mathrm{e}^{\mathrm{i} p \cdot x / \varepsilon^{s}}\left[\widehat{R}\left(\varepsilon^{s+\gamma} \omega, p\right)\left(1-\frac{\mathrm{e}^{i \omega h}+\mathrm{e}^{-i \omega h}}{2}\right) \frac{1}{\omega^{2}}\right]^{1 / 2},
$$

where $G$ is a real Gaussian field, symmetric in both variables $\omega$ and $p$, and such that $\mathbb{E}\left[G\left(\mathrm{~d} \omega_{1}, \mathrm{~d} p_{1}\right) G\left(\mathrm{~d} \omega_{2}, \mathrm{~d} p_{2}\right)\right]=$ $\delta\left(\omega_{1}-\omega_{2}\right) \delta\left(p_{1}-p_{2}\right)$. It then suffices to verify that $\mathbb{E}\left[\mathcal{B}_{m, h}^{\varepsilon}(x) \mathcal{B}_{n, h}^{\varepsilon}(y)\right]$ gives the appropriate correlation function following (1.4). 


\subsection{The phase modulation regime}

We suppose here that the initial wavefunction reads

$$
\phi_{0}^{\varepsilon}(x)=\phi_{0}\left(\varepsilon^{s_{c}} x\right), \quad \text { so that after rescaling } \quad \phi^{\varepsilon}(0, x)=\phi_{0}^{\varepsilon}\left(x / \varepsilon^{s}\right)=\phi_{0}\left(x / \varepsilon^{s-s_{c}}\right), \quad s_{c} \in[0, s] .
$$

We introduced the parameter $s_{c}$ to account for possible low frequency oscillations of the wavefunction. It is not a crucial point in this regime, but will be in the wave decoherence regime in the next section. The asymptotic limit of (1.3) was investigated in ([2], Thm. 1.2) and the authors use the following filtered wavefunction

$$
\zeta_{\kappa_{\gamma}}^{\varepsilon}(t, k)=\frac{1}{\varepsilon^{d\left(s-s_{c}\right)}} \widehat{\phi}^{\varepsilon}\left(t, \frac{k}{\varepsilon^{s-s_{c}}}\right) \mathrm{e}^{\mathrm{i}|k|^{2} t /\left(2 \varepsilon^{s-2 s_{c}}\right)}, \quad \text { with } \quad s=1 /\left(2 \kappa_{\gamma}\right), \text { and } s_{c} \in[0, s],
$$

where $\phi^{\varepsilon}$ satisfies (1.3) with initial data (2.5), and with

$$
\kappa_{0}=\frac{\alpha+2 \beta-1}{2 \beta} \in(1 / 2,1), \quad \text { and } \quad \kappa_{\gamma}=\frac{\kappa_{0}}{1-\gamma\left(\frac{\alpha+\beta-1}{\beta}\right)} \quad \text { for } \gamma \in[0,1) .
$$

The authors show that $\zeta_{\kappa_{\gamma}}^{\varepsilon}$ converges pointwise in $(t, k)$ in distribution to $\zeta(t, k)$ defined by (2.9) below. We then follow the same route here and consider the discrete version

$$
\zeta_{n}^{\varepsilon}(k)=\frac{1}{\varepsilon^{d\left(s-s_{c}\right)}} \widehat{\Psi}_{n}^{\varepsilon}\left(\frac{k}{\varepsilon^{s-s_{c}}}\right) \mathrm{e}^{\mathrm{i} n h \frac{|k|^{2}}{2 \varepsilon^{s-2 s_{c}}}},
$$

where $\widehat{\Psi}_{n}^{\varepsilon}(k)$ stands for the Fourier transform of $\Psi_{n}^{\varepsilon}(x)$ defined by (1.5). We have the following result.

Theorem 2.1. The process $\zeta_{n}^{\varepsilon}(k)$ defined by (2.8) converges pointwise in $k \in \mathbb{R}^{d}$ and in distribution as $\varepsilon \rightarrow 0$ to

$$
\zeta(n h, k)=\widehat{\phi}_{0}(k) \exp \left(\mathrm{i} \sqrt{D_{n, h}(k)} B_{\kappa_{0}}(n h)\right),
$$

for each $n \geq 0, h>0$ and $s_{c} \in[0, s]$, where $\left(B_{\kappa_{0}}(t)\right)_{t \geq 0}$ is a standard fractional Brownian motion with Hurst index $\kappa_{0}$ and $D_{n, h}$ is equal to

$$
D_{n, h}(k)=D=\frac{a(0) \Omega_{d}}{(2 \pi)^{d} \kappa_{0}\left(2 \kappa_{0}-1\right)} \int_{0}^{+\infty} \mathrm{d} \rho \frac{\mathrm{e}^{-\mu \rho^{2 \beta}}}{\rho^{2 \alpha-1}} \quad \text { if } \beta<\frac{1}{2} \text { or } \gamma>0,
$$

and verifies in the case $\beta=\frac{1}{2}$ and $\gamma=0$, for some $T>0$ :

$$
\lim _{h \rightarrow 0} D_{[T / h], h}(k)=D_{0}(k)=\frac{a(0)}{(2 \pi)^{d} \kappa_{0}\left(2 \kappa_{0}-1\right)} \int_{0}^{+\infty} \mathrm{d} \rho \frac{\mathrm{e}^{-\mu \rho}}{\rho^{2 \alpha-1}} \int_{\mathbb{S}^{d-1}} \mathrm{~d} S(u) \mathrm{e}^{\mathrm{i}|k| \rho u \cdot e_{1}},
$$

where [.] denotes integer part, $\Omega_{d}$ is the surface area of the unit sphere $\mathbb{S}^{d-1}$, and $\boldsymbol{e}_{1} \in \mathbb{S}^{d-1}$.

Theorem 2.1 shows that the splitting scheme captures the correct behavior as $\varepsilon \rightarrow 0$ for any stepsize $h>0$ independent of $\varepsilon$ when $\beta<\frac{1}{2}$ or $\gamma>0$. When $\beta=\frac{1}{2}$ and $\gamma=0$, the result has to interpreted as follows: pick a time $T>0$ and a let $n_{h}=[T / h]$. Then as $h \rightarrow 0$, independently of $\varepsilon, \zeta_{n_{h}}^{\varepsilon}(k)$ converges in law to $\zeta(T, k)=\widehat{\phi}_{0}(k) \exp \left(\mathrm{i} \sqrt{D_{0}(k)} B_{\kappa_{0}}(T)\right)$. As explained at the end of the introduction, such a result holds because we are only interested here in statistical quantities of the wavefunction. If we were interested in capturing trajectories, and not averages, we would need to satisfy the error estimate (1.6) which is much more stringent. In a statistical sense, the Laplacian and the potential do not interact with each other, which explains the absence of constraint on $h$. The limit $\varepsilon \rightarrow 0$ then takes care of the convergence to the fractional Brownian motion in the potential term. In the case $\beta<\frac{1}{2}$ or $\gamma>0$, we recover the limit of $\zeta$ for any $h>0$; when $\beta=\frac{1}{2}$ and $\gamma=0$, we need in addition $h \rightarrow 0$. In the latter case, the oscillations of the potential are not fast enough to directly recover the exact behavior, one needs $h \ll 1$. The next section is devoted to regime (ii). 


\subsection{Wave decoherence regime}

This regime arises for moderate distances propagation of order $\varepsilon^{-s}$, for some $s \in\left(1 /\left(2 \kappa_{\gamma}\right), 1\right)$ that depends on the random medium and the oscillations of the initial condition. The parameter $\kappa_{\gamma}$ is defined in (2.7). The denomination for this regime stems from the fact that a diffusion in momentum takes place at such distances of propagation, see [9] for more details. It is particular to the long-range correlation case, and does not exist in the short-range situation. The wave decoherence effect is observed on the Wigner transform of the wavefunction provided the initial condition has sufficiently low frequency oscillations. The Wigner transform is a classical tool in high frequency analysis, see [12] for more details. It is customary in this context to consider Wigner transforms of mixed states wavefunctions, which provide uniform bounds in $L^{2}$ instead of pure states wavefunctions which only offer bounds in some distribution spaces [12]. We therefore consider an initial condition of the form

$$
\phi^{\varepsilon}(0, x, \zeta)=\phi_{0}(x) \exp \left(\mathrm{i} \zeta \cdot x / \varepsilon^{s-s_{c}}\right)
$$

where $\zeta \in \mathbb{R}^{d}$ is the mixed states parameter and $s_{c} \in(0, s]$ is here to account for some low frequency oscillations that are necessary to observe the wave decoherence effects. Such effects arise at distances $\varepsilon^{-s}$ with $s=1-s_{c} \theta$, where

$$
\theta=2(\alpha+\beta-1) \in(0,1)
$$

is a parameter that only depends on the correlation range of the random medium. Note that the radiative transfer regime holds at distances of order $\varepsilon^{-1}$, so that one needs $s_{c}>0$ for the wave decoherence to take place. Remark that $s_{c} \leq s$ implies $s_{c} \leq 1 /(1+\theta)$, and consequently that $s \geq 1 /(1+\theta)>1 /\left(2 \kappa_{\gamma}\right)$ for the parameters $\alpha, \beta, \gamma$ and $\theta$ defined in (2.4)-(2.13). There is a critical case when $s_{c}=s=1 /(1+\theta)$ addressed in Theorem 2.3.

Since the initial condition oscillates at a frequency $\varepsilon^{s_{c}-s}$, it is natural to consider Wigner transforms correlating wavefunctions at a scale $\varepsilon^{s-s_{c}}$, whence the following definition:

$$
W^{\varepsilon}(t, x, k)=\frac{1}{(2 \pi)^{d}} \int_{\mathbb{R}^{d} \times S} \mathrm{~d} y \nu(\mathrm{d} \zeta) \mathrm{e}^{\mathrm{i} k \cdot y} \phi^{\varepsilon}\left(t, x-\varepsilon^{s-s_{c}} \frac{y}{2}, \zeta\right) \overline{\phi^{\varepsilon}\left(t, x+\varepsilon^{s-s_{c}} \frac{y}{2}, \zeta\right)},
$$

where $(S, \mathcal{S}, \nu(\mathrm{d} \zeta))$ is a probability space. The weak limit in $L^{2}$ of $W^{\varepsilon}(t=0)$ therefore reads:

$$
W_{0}(x, k)=\left\{\begin{array}{l}
\left|\phi_{0}(x)\right|^{2} \widehat{\nu}(k), \quad \text { if } s_{c}<s, \\
\frac{1}{(2 \pi)^{d}} \int_{\mathbb{R}^{d} \times S} \mathrm{~d} y \nu(\mathrm{d} \zeta) \mathrm{e}^{\mathrm{i}(k-\zeta) \cdot y} \phi_{0}(x-y / 2) \overline{\phi_{0}(x+y / 2)}, \quad \text { if } s=s_{c} .
\end{array}\right.
$$

Note that the limit actually holds in the strong sense since $\left\|W^{\varepsilon}(0)\right\|_{L^{2}} \rightarrow\left\|W_{0}\right\|_{L^{2}}$. It is then well-known that the $L^{2}$ norm of the Wigner transform is conserved [12]:

$$
\left\|W^{\varepsilon}(t)\right\|_{L^{2}\left(\mathbb{R}^{2 d}\right)}=\left\|W^{\varepsilon}(0)\right\|_{L^{2}\left(\mathbb{R}^{2 d}\right.} \leq C
$$

where $C$ is independent of $\varepsilon$. 
The limiting Wigner transform essentially exhibits three different behaviors [9]. When $0<s_{c}<s$, the Wigner transform is self-averaging and converges in probability weakly in $L^{2}$ to the solution to the fractional heat equation (2.18); when $s=s_{c}$, two situations arises: if $\beta \neq \frac{1}{2}$, or $\gamma \neq 0$, the Wigner transform converges in distribution strongly in $L^{2}$ to the stochastic fractional heat equation (2.20); the case $\beta=\frac{1}{2}$ and $\gamma=0$ is an end point for Theorem 2.1 and there is consequently no wave decoherence. In order to capture these phenomena using the time-splitting scheme (1.5), we consider the following discretized version of the Wigner transform:

$$
W_{n, h}^{\varepsilon}(x, k)=\frac{1}{(2 \pi)^{d}} \int_{\mathbb{R}^{d} \times S} \mathrm{~d} y \nu(\mathrm{d} \zeta) \mathrm{e}^{\mathrm{i} k \cdot y} \Psi_{n}^{\varepsilon}\left(x-\varepsilon^{s-s_{c}} \frac{y}{2}, \zeta\right) \overline{\Psi_{n}^{\varepsilon}\left(x+\varepsilon^{s-s_{c}} \frac{y}{2}, \zeta\right)},
$$

where $\Psi_{n}^{\varepsilon}(x)$ is defined by (1.5) with initial condition (2.12). We have the following result:

Theorem 2.2. Let $s=1-\theta s_{c}$, where $\theta$ is defined in (2.13) and $s_{c} \in(0, s)$. Then, we have for all $n>0, h>0$, $\eta>0$, and all test function $\varphi \in L^{2}\left(\mathbb{R}^{2 d}\right)$ :

$$
\lim _{\varepsilon \rightarrow 0} \mathbb{P}\left(\left|\left\langle W_{n, h}^{\varepsilon}-W(n h), \varphi\right\rangle_{L^{2}\left(\mathbb{R}^{2 d}\right)}\right|>\eta\right)=0, \quad \text { where } \quad W(t, x, k)=\frac{1}{(2 \pi)^{d}} \int_{\mathbb{R}^{d}} \mathrm{~d} q \mathrm{e}^{\mathrm{i} k \cdot q-\sigma(\theta)|q|^{\theta} t} \widehat{W}_{0}^{k}(x, q),
$$

which is the unique solution uniformly bounded in $L^{2}\left(\mathbb{R}^{2 d}\right)$ of

$$
\partial_{t} W=-\sigma(\theta)\left(-\Delta_{k}\right)^{\theta / 2} W, \quad \text { with } \quad \sigma(\theta)=\frac{2 a(0) \theta \Gamma(1-\theta)}{\mu(2 \pi)^{d}} \int_{\mathbb{S}^{d-1}} \mathrm{~d} S(u)\left|\boldsymbol{e}_{1} \cdot u\right|^{\theta},
$$

where $\left(-\Delta_{k}\right)^{\theta / 2}$ is the fractional Laplacian with Hurst index $\theta \in(0,1), e_{1} \in \mathbb{S}^{d-1}$, and $\Gamma(z)=\int_{0}^{+\infty} t^{1-z} \mathrm{e}^{-t} \mathrm{~d} t$. In (2.17), $\widehat{W}_{0}^{k}$ stands for the Fourier transform with respect to the variable $k$ of the limiting initial condition $W_{0}$ defined by $(2.15)$.

In this regime too, the splitting scheme captures the correct asymptotic behavior for any $h>0$. The limiting $W$ is deterministic, so that the loss of coherence does not depend on the particular realization of the potential. The spatial parameter $x$ is frozen, so that the density $n(t, x)=\int_{\mathbb{R}^{d}} W(t, x, k) \mathrm{d} k$ is equal to $n(0, x)$. It is a consequence of the fact that dispersion is of order $\varepsilon^{s_{c}}$, which is small compared to the momentum diffusion mechanism of order one. The interaction between the Laplacian (or the transport term in the Wigner equation) is negligible, which explains why there are no constraints on the stepsize $h$. The dispersion can be captured by the Wigner transform when $s_{c}=0$ and $s=1$, which is the object of Section 2.3.

The following result concerns the critical case $s_{c}=s=1 /(1+\theta)$, with either $\gamma \neq 0$ or $\beta \neq 1 / 2$. As already explained, the remaining case $s=s_{c}, \gamma=0$ and $\beta=1 / 2$ is included in Theorem 2.1.

Theorem 2.3. For either $\gamma>0$ or $\beta<1 / 2$, and $s_{c}=s=1 /(1+\theta)$, where $\theta \in(0,1)$ is defined by (2.13), and for all $n>0$ and $h>0,\left(W_{n, h}^{\varepsilon}\right)_{\varepsilon \in(0,1)}$ defined by (2.16) converges in distribution on $L^{2}\left(\mathbb{R}^{2 d}\right)$, equipped with the strong topology, as $\varepsilon \rightarrow 0$ to a limit $W(n h, x, k)$ defined by

$$
W(t, x, k)=\frac{1}{(2 \pi)^{d}} \int_{\mathbb{R}^{d}} \mathrm{~d} q \widehat{W}_{0}^{k}(x, q) \exp \left(\mathrm{i} k \cdot q+\mathrm{i} \int_{\mathbb{R}^{d}} \mathcal{B}_{t}(d p) \mathrm{e}^{\mathrm{i} p \cdot x}\left(\mathrm{e}^{-\mathrm{i} q \cdot p / 2}-\mathrm{e}^{\mathrm{i} q \cdot p / 2}\right)\right),
$$

which is the unique solution of the stochastic fractional heat equation

$$
\begin{aligned}
d W(t, x, k)= & -\sigma(\theta)\left(-\Delta_{k}\right)^{\theta / 2} W(t, x, k) \\
& +i \int_{\mathbb{R}^{d}} \mathrm{~d} \mathcal{B}_{t}(\mathrm{~d} p) \mathrm{e}^{\mathrm{i} x \cdot p}\left(W\left(t, x, k-\frac{p}{2}\right)-W\left(t, x, k+\frac{p}{2}\right)\right),
\end{aligned}
$$


where $\left(-\Delta_{k}\right)^{\theta / 2}$ is the fractional Laplacian with Hurst index $\theta \in(0,1), \sigma(\theta)$ is defined by $(2.18)$, and $\left(\mathcal{B}_{t}\right)_{t}$ is a real Brownian motion on the dual space of

$$
\mathcal{H}_{\theta}=\left\{\varphi \text { such that } \varphi(p)=\overline{\varphi(-p)} \text { and } \int_{\mathbb{R}^{d}} \frac{\mathrm{d} p}{|p|^{d+\theta}}|\varphi(p)|^{2}<+\infty\right\}
$$

with covariance function

$$
\mathbb{E}\left[\mathcal{B}_{t}(\varphi) \mathcal{B}_{s}(\psi)\right]=s \wedge t \frac{2 a(0)}{\mu(2 \pi)^{d}} \int_{\mathbb{R}^{d}} \frac{\mathrm{d} p}{|p|^{d+\theta}} \varphi(p) \overline{\psi(p)}, \quad \forall(\varphi, \psi) \in \mathcal{H}_{\theta} \times \mathcal{H}_{\theta}
$$

In (2.19), $\widehat{W}_{0}^{k}$ stands for the Fourier transform of $W_{0}$ defined by (2.15) with respect to the variable $k$.

The splitting scheme captures again the correct asymptotics for any $h>0$. In the critical case, the limiting Wigner transform is random because the wave propagates over shorter distances than in Theorem 2.2 that are not sufficient to average out the stochastic effects. Indeed, in Theorem 2.2, waves propagate over a distance $\varepsilon^{-s}$ with $s>1 /(1+\theta)$ (since $s_{c}<s$ ) while they propagate over $\varepsilon^{-1 /(1+\theta)}$ in Theorem 2.3. As shown in [9], the convergence holds in $L^{2}\left(\mathbb{R}^{2 d}\right)$ strong in the critical case since the $L^{2}$ norm is conserved: $\|W(t)\|_{L^{2}\left(\mathbb{R}^{2 d}\right)}=\left\|W_{0}\right\|_{L^{2}\left(\mathbb{R}^{2 d}\right)}$. The next section is devoted to the radiative transfer regime.

\subsection{Radiative transfer regime}

This section considers the radiative transfer limit with $s=1$ and $s_{c}=0$ obtained in [8] for $\gamma>0$. We show that the time-splitting scheme (1.5) for a stepsize $h \ll 1$, independent of $\varepsilon$, leads to the correct limit. More precisely, we have the following result:

Theorem 2.4. For $\gamma>0$, for all $n>0, h>0, \eta>0$, and all test function $\varphi \in L^{2}\left(\mathbb{R}^{2 d}\right)$, we have

$\lim _{\varepsilon \rightarrow 0} \mathbb{P}\left(\left|\left\langle W_{n, h}^{\varepsilon}-W_{n, h}, \varphi\right\rangle_{L^{2}\left(\mathbb{R}^{2 d}\right)}\right|>\eta\right)=0, \quad$ where $\quad W_{n, h}(x, k)=\frac{1}{(2 \pi)^{2 d}} \int \mathrm{d} y \mathrm{~d} q \mathrm{e}^{\mathrm{i}(x \cdot y+k \cdot q)+\psi_{n, h, \theta}(y, q)} \widehat{W}_{0}(y, y+t q)$,

with

$$
\psi_{n, h, \theta}(y, q)=h \sum_{j=1}^{n} \psi(y+n h(1-j / n+1 / n) q),
$$

so that $\lim _{h \rightarrow 0} W_{n, h}(x, k)=W(t, x, k)$ with

$$
W(t, x, k)=\frac{1}{(2 \pi)^{2 d}} \int_{\mathbb{R}^{2 d}} \mathrm{~d} y \mathrm{~d} q \mathrm{e}^{\mathrm{i}(x \cdot q+k \cdot y)+\int_{0}^{t} \mathrm{~d} u \psi(y+u q)} \widehat{W}_{0}(q, y+t q),
$$

and

$$
\psi(q)=\frac{2}{\mu(2 \pi)^{d}} \int_{\mathbb{R}^{d}} \mathrm{~d} p \frac{a(p)}{|p|^{d+\theta}}\left(\mathrm{e}^{\mathrm{i} p \cdot q}-1\right),
$$

which is the unique solution uniformly bounded in $L^{2}\left(\mathbb{R}^{2 d}\right)$ of

$$
\partial_{t} W(t, x, k)+k \cdot \nabla_{x} W(t, x, k)=\frac{2}{\mu(2 \pi)^{d}} \int_{\mathbb{R}^{d}} \mathrm{~d} p \frac{a(p)}{|p|^{d+\theta}}(W(t, x, k+p)-W(t, x, k)) .
$$

In (2.21), $\widehat{W}_{0}$ stands for the Fourier transform in both variables $x$ and $k$ of $W_{0}$ defined by (2.15). 
This case is different than the one of Section 2.2 since transport is now of order one and of same order as momentum diffusion. The fact that transport has no influence on the expression of the diffusion term in the limit $\varepsilon \rightarrow 0$, allowing for a stepsize $h$ independent of $\varepsilon$, is due to the fast oscillations in time of the potential when $\gamma>0$. When $\gamma=0$, the situation is different and transport and diffusion interact to lead to another expression of the collision operator, which is

$$
\mathcal{L} \varphi(k)=\int_{\mathbb{R}^{d}} \mathrm{~d} p \sigma\left(p-k, \frac{|k|^{2}-|p|^{2}}{2}\right)(\varphi(p)-\varphi(k)) \quad \text { and } \quad \sigma(p, \omega)=\frac{2 \mu|p|^{2 \beta} a(p)}{(2 \pi)^{d}|p|^{d+2(\alpha-1)}\left(\mu^{2}|p|^{4 \beta}+\omega^{2}\right)} .
$$

This is very similar to what was observed in [3] for the short-range case. When $\gamma=0$, which is the scaling considered in [3], one needs $h=\varepsilon h^{\prime}$, with $h^{\prime} \ll 1$ independent of $\varepsilon$ to recover the correct collision operator. The same situation holds for the long-range case when $\gamma=0$ : one needs $h=f(\varepsilon) h^{\prime}$, for some function $f(x) \rightarrow 0$ (that has to be determined) as $\varepsilon \rightarrow 0$. The case $\gamma>0$ is similar to the white-noise case mentioned in [3] for which there are no $\varepsilon$-dependent constraint on $h$. The corresponding collision operator is

$$
\mathcal{L} \varphi(k)=\int_{\mathbb{R}^{d}} \mathrm{~d} p \sigma(p-k, 0)(\varphi(p)-\varphi(k))
$$

which does not depend on $|k|^{2}-\left|p^{2}\right|^{2}$, the latter term being an outcome of the interaction between transport and diffusion. The remaining part of the paper is devoted to the Proofs of Theorems 2.1-2.4.

\section{Proof of Theorem 2.1}

The outline of the proof is as follows: we compute the limits of all moments of the form $\mathbb{E}\left(\zeta_{n}^{\varepsilon}(k)^{M}\left(\zeta_{n}^{\varepsilon}(k)^{*}\right)^{N}\right)$, for $M, N \geq 0$ when $\varepsilon \rightarrow 0$. Since in particular the second order moment converges, the sequence of distributions of $\left(\zeta_{n}^{\varepsilon}(k)\right)_{n}$ is tight in $\mathbb{C}$. In order to identify the accumulation points, we show that the limiting distribution is unique and determined by its moments using Carleman's criterion for the moment problem as in [2]. This then gives the convergence in distribution to the $\zeta$ defined by (2.9). Notice that compared to the continuous version of the problem in [2], the proof here is much more direct since there are no diagrammatic expansions involved. In [2], one has to estimate expectations of product of potentials $V$ at different points and related pairings. This difficulty is not present in our computations due to the structure of the splitting scheme: there are no products of potentials, but products of complex exponentials of potentials, which simplifies to the exponential of the corresponding sum of second order moments. The expectation of such an exponential is then straightforward since the potential $V$ is Gaussian.

We start by computing the Fourier transform in $x$ of $\Psi_{n}^{\varepsilon}$, which reads

$$
\widehat{\Psi}_{n}^{\varepsilon}(k)=\mathrm{e}^{-\mathrm{i} \varepsilon^{s} h \frac{|k|^{2}}{2}}(2 \pi)^{-d} \int_{\mathbb{R}^{d}} \int_{\mathbb{R}^{d}} \mathrm{~d} p_{n} \mathrm{~d} x_{n} \mathrm{e}^{-\mathrm{i}\left(k-p_{n}\right) \cdot x_{n}} \mathrm{e}^{-\mathrm{i} \mathcal{B}_{\varepsilon}^{n}\left(x_{n}\right)} \widehat{\Psi}_{n-1}^{\varepsilon}\left(p_{n}\right) .
$$

For the function $\zeta_{n}^{\varepsilon}(k)$, rescaling $p_{n}$ by $p_{n} / \varepsilon^{s-s_{c}}$, this translates into

$$
\zeta_{n}^{\varepsilon}(k)=\frac{\mathrm{e}^{\mathrm{i}(n-1) h \frac{|k|^{2}}{2 \varepsilon^{s-2 s_{c}}}}}{(2 \pi)^{d} \varepsilon^{d\left(s-s_{c}\right)}} \int_{\mathbb{R}^{d}} \int_{\mathbb{R}^{d}} \mathrm{~d} x_{n} \mathrm{~d} p_{n} \mathrm{e}^{-\mathrm{i}\left(k-p_{n}\right) \cdot x_{n} / \varepsilon^{s-s_{c}}} \mathrm{e}^{-\mathrm{i} \mathcal{B}_{\varepsilon}^{n}\left(x_{n}\right)} \mathrm{e}^{-\mathrm{i}(n-1) h \frac{\left|p_{n}\right|^{2}}{2 \varepsilon^{s-2 s_{c}}} \zeta_{n-1}^{\varepsilon}}\left(p_{n}\right) .
$$

Introducing the notation

$$
\int_{\mathbb{R}^{d}} \mathrm{~d} x_{1} \cdots \int_{\mathbb{R}^{d}} \mathrm{~d} x_{n} \equiv \int \mathrm{d} X, \quad \int_{\mathbb{R}^{d}} \mathrm{~d} p_{1} \cdots \int_{\mathbb{R}^{d}} \mathrm{~d} p_{n} \equiv \int \mathrm{d} P,
$$

with $X=\left(x_{1}, \ldots, x_{n}\right) \in \mathbb{R}^{n d}, P=\left(p_{1}, \ldots, p_{n}\right) \in \mathbb{R}^{n d}, x_{i} \in \mathbb{R}^{d}, p_{i} \in \mathbb{R}^{d}, i=1, \ldots, n$, we have

$$
\zeta_{n}^{\varepsilon}(k)=\frac{\mathrm{e}^{\mathrm{i}(n-1) h \frac{|k|^{2}}{2 \varepsilon^{s-2 s_{c}}}}}{(2 \pi)^{n d} \varepsilon^{n d\left(s-s_{c}\right)}} \int \mathrm{d} X \mathrm{~d} P A_{\varepsilon}(X, P) B_{\varepsilon}(X) \zeta_{0}^{\varepsilon}\left(p_{1}\right),
$$


where

$$
A_{\varepsilon}(X, P)=\mathrm{e}^{-\mathrm{i} \frac{h}{2 \varepsilon^{s-2 s_{c}}} \sum_{j=2}^{n}\left|p_{j}\right|^{2}} \mathrm{e}^{-\mathrm{i} \sum_{j=1}^{n}\left(p_{j+1}-p_{j}\right) \cdot x_{j} / \varepsilon^{s-s_{c}}} \quad \text { and } \quad B_{\varepsilon}(X)=\mathrm{e}^{-\mathrm{i} \sum_{j=1}^{n} \mathcal{B}_{\varepsilon}^{j}\left(x_{j}\right)},
$$

with $p_{n+1}=k$. For the sake of clarity, we investigate first the limit of the expectation of $\zeta_{n}^{\varepsilon}$.

\subsection{Convergence of the expectation}

Since $V$ is a Gaussian process, we have

$$
\mathbb{E}\left\{B_{\varepsilon}(X)\right\}=\mathrm{e}^{-\frac{1}{2} \operatorname{Var}\left(\sum_{j=1}^{n} \mathcal{B}_{\varepsilon}^{j}\left(x_{j}\right)\right)}
$$

where, according to (2.1):

$$
v_{\varepsilon}(X):=\operatorname{Var}\left(\sum_{j=1}^{n} \mathcal{B}_{\varepsilon}^{j}\left(x_{j}\right)\right)=\frac{1}{\varepsilon^{2 s-(1-\gamma)}} \sum_{j, l=1}^{n} \int_{(j-1) h}^{j h} \int_{(l-1) h}^{l h} R\left(\frac{u_{1}-u_{2}}{\varepsilon^{s+\gamma}}, \frac{x_{j}-x_{i}}{\varepsilon^{s}}\right) \mathrm{d} u_{1} \mathrm{~d} u_{2} .
$$

After the change of variables $k \rightarrow k \varepsilon^{\frac{s+\gamma}{2 \beta}}$, we find

$$
R\left(\frac{t}{\varepsilon^{s+\gamma}}, \frac{x}{\varepsilon^{s}}\right)=\frac{\varepsilon^{\frac{1-\alpha}{\beta}}}{(2 \pi)^{d}} \int_{\mathbb{R}^{d}} \mathrm{~d} k \mathrm{e}^{\mathrm{i} \varepsilon^{\frac{s+\gamma}{2 \beta}-s} k \cdot x} \mathrm{e}^{-\mu|k|^{2 \beta}} \frac{a\left(\varepsilon^{\frac{s+\gamma}{2 \beta}} k\right)}{|k|^{2 \alpha+d-2}}:=\varepsilon^{\frac{(1-\alpha)(s+\gamma)}{\beta}} R_{\varepsilon}\left(t, \varepsilon^{\frac{s+\gamma}{2 \beta}-s} x\right) .
$$

This yields

$$
v_{\varepsilon}(X)=\varepsilon^{\frac{(1-\alpha)(s+\gamma)}{\beta}-2 s+1-\gamma} \sum_{j, l=1}^{n} \int_{(j-1) h}^{j h} \int_{(l-1) h}^{l h} R_{\varepsilon}\left(u_{1}-u_{2}, \varepsilon^{\frac{s+\gamma}{2 \beta}-s}\left(x_{j}-x_{i}\right)\right) \mathrm{d} u_{1} \mathrm{~d} u_{2} .
$$

Since $s=\frac{1}{2 \kappa_{\gamma}}$, the power of $\varepsilon$ above is equal to one. Going back to $\zeta_{n}^{\varepsilon}$, and making the change of variables

$$
p_{j} \rightarrow \varepsilon^{s-s_{c}} p_{j}+p_{j+1}, \quad \text { so that } \quad p_{j} \rightarrow \varepsilon^{s-s_{c}} \sum_{i=j}^{n} p_{i}+k:=\varepsilon^{s-s_{c}} \tilde{p}_{j}+k, \quad j=1, \ldots, n,
$$

we find

$$
\mathbb{E}\left\{\zeta_{n}^{\varepsilon}(k)\right\}=(2 \pi)^{-n d} \int \mathrm{d} X \mathrm{~d} P \mathrm{e}^{-\mathrm{i} \frac{\varepsilon^{s} h}{2} \sum_{j=2}^{n}\left|\tilde{p}_{j}\right|^{2}} \mathrm{e}^{-\mathrm{i} \varepsilon^{s_{c}} h k \cdot \sum_{j=1}^{n} \tilde{p}_{j}} \mathrm{e}^{\mathrm{i} \sum_{j=1}^{n} p_{j} \cdot x_{j}} \mathrm{e}^{-\frac{1}{2} v_{\varepsilon}(X)} \zeta_{0}^{\varepsilon}\left(\varepsilon^{s-s_{c}} \tilde{p}_{1}+k\right) .
$$

The limit of $\mathbb{E}\left\{\zeta_{n}^{\varepsilon}(k)\right\}$ depends on $\beta$ and $\gamma$, and yields two different expressions. We treat both limits separately.

Case $\beta<\frac{1}{2}$ or $\gamma>\mathbf{0}$. In such case, $\frac{s+\gamma}{2 \beta}-s>0$ so that the limit of $v_{\varepsilon}$ does not depend on $X$. Passing formally to the limit in (3.1), using the fact that $\zeta_{0}^{\varepsilon}\left(\varepsilon^{s-s_{c}} \tilde{p}_{1}+k\right)=\widehat{\phi}_{0}\left(\varepsilon^{s-s_{c}} \tilde{p}_{1}+k\right)$, and defining $\delta\left(s_{c}\right)=0$ if $s_{c}>0$ and $\delta\left(s_{c}\right)=1$ if $s_{c}=0$, it comes

$$
\begin{aligned}
\mathbb{E}\left\{\zeta_{n}^{\varepsilon}(k)\right\} & \rightarrow \mathrm{e}^{-v_{0} / 2}(2 \pi)^{-n d} \int \mathrm{d} X \mathrm{~d} P \mathrm{e}^{-\mathrm{i} \delta\left(s_{c}\right) h k \cdot \sum_{j=1}^{n} \tilde{p}_{j}} \mathrm{e}^{\mathrm{i} \sum_{j=1}^{n} p_{j} \cdot x_{j}} \widehat{\phi}_{0}\left(\delta\left(s-s_{c}\right) \tilde{p}_{1}+k\right) \\
& =\mathrm{e}^{-v_{0} / 2} \int \mathrm{d} P \mathrm{e}^{-\mathrm{i} \delta\left(s_{c}\right) h k \cdot \sum_{j=1}^{n} \tilde{p}_{j}} \widehat{\phi}_{0}\left(\delta\left(s-s_{c}\right) \tilde{p}_{1}+k\right) \prod_{j=1}^{n} \delta\left(p_{j}\right) \\
& =\mathrm{e}^{-v_{0} / 2} \widehat{\phi}_{0}(k),
\end{aligned}
$$


with

$$
\begin{aligned}
v_{0}:=\lim _{\varepsilon \rightarrow 0} v_{\varepsilon}(X) & =\int_{0}^{n h} \int_{0}^{n h} \mathrm{~d} s \mathrm{~d} u R_{0}(u-s, 0)=\frac{a(0)}{(2 \pi)^{d}} \int_{0}^{n h} \int_{0}^{n h} \int_{\mathbb{R}^{d}} \mathrm{~d} s \mathrm{~d} u \mathrm{~d} k \frac{\mathrm{e}^{-\mu|k|^{2 \beta}|s-u|}}{|k|^{2 \alpha+d-2}} \\
& =\frac{a(0)}{(2 \pi)^{d}} \int_{0}^{n h} \int_{0}^{n h} \mathrm{~d} s \mathrm{~d} u|u-s|^{2 \kappa_{0}-2} \int_{\mathbb{R}^{d}} \mathrm{~d} k \frac{\mathrm{e}^{-\mu|k|^{2 \beta}}}{|k|^{2 \alpha+d-2}} \\
& =\frac{a(0)(n h)^{2 \kappa_{0}}}{(2 \pi)^{d} \kappa_{0}\left(2 \kappa_{0}-1\right)} \int_{\mathbb{R}^{d}} \mathrm{~d} k \frac{\mathrm{e}^{-\mu|k|^{2 \beta}}}{|k|^{2 \alpha+d-2}}=(n h)^{2 \kappa_{0}} D,
\end{aligned}
$$

where $D$ si defined in (2.10).

Case $\boldsymbol{\beta}=\frac{1}{2}, \boldsymbol{\gamma}=\mathbf{0}$. Recast first $\widehat{\phi}_{0}\left(\delta\left(s-s_{c}\right) \tilde{p}_{1}+k\right)$ as $\widehat{\phi}_{0}\left(\delta\left(s-s_{c}\right) \tilde{p}_{1}+k\right)=\int \mathrm{e}^{-\mathrm{i} x \cdot\left(\delta\left(s-s_{c}\right) \tilde{p}_{1}+k\right)} \phi_{0}(x) \mathrm{d} x$. Passing formally to the limit in (3.1) yields

$$
\mathbb{E}\left\{\zeta_{n}^{\varepsilon}(k)\right\} \rightarrow(2 \pi)^{-n d} \int \mathrm{d} X \mathrm{~d} P \mathrm{~d} x \mathrm{e}^{-\mathrm{i} \delta\left(s_{c}\right) h k \cdot \sum_{j=1}^{n} \tilde{p}_{j}} \mathrm{e}^{\mathrm{i} \sum_{j=1}^{n} p_{j} \cdot\left(x_{j}-\delta\left(s-s_{c}\right) x\right)} \mathrm{e}^{-\frac{1}{2} v_{0}(X)} \mathrm{e}^{-\mathrm{i} x \cdot k} \phi_{0}(x),
$$

where

$$
v_{0}(X)=\sum_{j, l=1}^{n} \int_{(j-1) h}^{j h} \int_{(l-1) h}^{l h} R_{0}\left(u_{1}-u_{2},\left(x_{j}-x_{i}\right)\right) \mathrm{d} u_{1} \mathrm{~d} u_{2}
$$

Realizing that

$$
\sum_{j=1}^{n} \tilde{p}_{j}=\sum_{j=1}^{n} j p_{j}
$$

we find

$$
\begin{aligned}
\int \mathrm{d} X \mathrm{~d} P \mathrm{e}^{-\mathrm{i} \delta\left(s_{c}\right) h k \cdot \sum_{j=1}^{n} \tilde{p}_{j}} \mathrm{e}^{\mathrm{i} \sum_{j=1}^{n} p_{j} \cdot\left(x_{j}-\delta\left(s-s_{c}\right) x\right)} \mathrm{e}^{-\frac{1}{2} v_{0}(X)} & =\int \mathrm{d} X \mathrm{~d} P \mathrm{e}^{\mathrm{i} \sum_{j=1}^{n} p_{j} \cdot\left(x_{j}-k j h \delta\left(s_{c}\right)\right)-\delta\left(s-s_{c}\right) x} \mathrm{e}^{-\frac{1}{2} v_{0}(X)} \\
& =(2 \pi)^{n d} \int \mathrm{d} X \prod_{j=1}^{n} \delta\left(x_{j}-k j h \delta\left(s_{c}\right)-\delta\left(s-s_{c}\right) x\right) \mathrm{e}^{-\frac{1}{2} v_{0}(X)}
\end{aligned}
$$

This implies that $\mathbb{E}\left\{\zeta_{n}^{\varepsilon}(k)\right\} \rightarrow \widehat{\phi}_{0}(k) \mathrm{e}^{-\frac{1}{2} v_{0}(n, h)}$ where

$$
v_{0}(n, h):=(n k)^{2 \kappa_{0}} D_{n, h}=\int_{0}^{h} \int_{0}^{h} \mathrm{~d} s \mathrm{~d} u \sum_{j=1}^{n} \sum_{l=1}^{n} R_{0}\left((j-l) h+u-s, k(j-l) h \delta\left(s_{c}\right)\right),
$$

and therefore does not depend on $\delta\left(s-s_{c}\right)$ any longer. When $s_{c}>0$, we recover $v_{0}(n, h)=(n h)^{\kappa_{0}} D$. When $s_{c}=0$, take $T>0$, and let $n=[T / h]$, where [.] denotes the integer part. Then, (3.4) is the Riemann sum of

$$
\begin{aligned}
\frac{1}{h^{2}} \int_{0}^{h} \int_{0}^{h} \mathrm{~d} s \mathrm{~d} u \int_{0}^{T} & \int_{0}^{T} \mathrm{~d} t \mathrm{~d} \tau \tilde{R}(t-\tau+u-s, k(t-\tau)) \\
& \rightarrow h \rightarrow 0 \int_{0}^{T} \int_{0}^{T} \mathrm{~d} t \mathrm{~d} \tau \tilde{R}(t-\tau, k(t-\tau))=\frac{a(0)(T)^{2 \kappa}}{(2 \pi)^{d} \kappa(2 \kappa-1)} \int_{\mathbb{R}^{d}} \mathrm{~d} q \frac{\mathrm{e}^{-\mu|q|^{2 \beta}} \mathrm{e}^{\mathrm{i} k \cdot q}}{|q|^{2 \alpha+d-2}}
\end{aligned}
$$

which is the expected limit. 


\subsection{Convergence of the moments}

We now look at the limit of $\mathbb{E}\left\{\left(\zeta_{n}^{\varepsilon}(k)\right)^{M}\left(\zeta_{n}^{\varepsilon}(k)^{*}\right)^{N}\right\}$. Computations are very similar to that of the expectation. The term $\zeta_{n}^{\varepsilon}(k)^{M}\left(\zeta_{n}^{\varepsilon}(k)^{*}\right)^{N}$ can be written as

$$
\left.\zeta_{n}^{\varepsilon}(k)\right)^{M}\left(\zeta_{n}^{\varepsilon}(k)^{*}\right)^{N}=\frac{\mathrm{e}^{\mathrm{i}(n-1)(M-N) h \frac{|k|^{2}}{2^{s-2 s_{c}}}}}{(2 \pi)^{(M+N) n d} \varepsilon^{(M+N) n d\left(s-s_{c}\right)}} \int \mathrm{d} X \mathrm{~d} P A_{\varepsilon}(X, P) B_{\varepsilon}(X) \widehat{\Phi}_{0}(P),
$$

where we used the notation

$$
\prod_{i=1}^{n} \prod_{j=1}^{M+N} \int_{\mathbb{R}^{d}} \mathrm{~d} x_{i}^{j} \equiv \int \mathrm{d} X, \quad \prod_{i=1}^{n} \prod_{j=1}^{M+N} \int_{\mathbb{R}^{d}} \mathrm{~d} p_{i}^{j} \equiv \mathrm{d} P
$$

with

$$
\begin{aligned}
X & =\left(x^{1}, \ldots, x^{M}, x^{M+1}, \ldots, x^{M+N}\right) \in \mathbb{R}^{(M+N) n d}, \quad x^{j}=\left(x_{1}^{j}, \ldots, x_{n}^{j}\right) \in \mathbb{R}^{n d}, \\
P & =\left(p^{1}, \ldots, p^{M}, p^{M+1}, \ldots, p^{M+N}\right) \in \mathbb{R}^{(M+N) n d}, \quad p^{j}=\left(p_{1}^{j}, \ldots, p_{n}^{j}\right) \in \mathbb{R}^{n d}, \quad p_{i}^{j} \in \mathbb{R}^{d},
\end{aligned}
$$

and

$$
\begin{aligned}
A_{\varepsilon}(X, P)= & \exp \left\{-\frac{\mathrm{i} h}{2 \varepsilon^{s-2 s_{c}}}\left(\sum_{j=1}^{M} \sum_{i=2}^{n}\left|p_{i}^{j}\right|^{2}-\sum_{j=M+1}^{M+N} \sum_{i=2}^{n}\left|p_{i}^{j}\right|^{2}\right)\right\} \\
& \times \exp \left\{-\frac{\mathrm{i}}{\varepsilon^{s-s_{c}}}\left(\sum_{j=1}^{M} \sum_{i=1}^{n}\left(p_{i+1}^{j}-p_{i}^{j}\right) \cdot x_{i}^{j}-\sum_{j=M+1}^{M+N} \sum_{i=1}^{n}\left(p_{i+1}^{j}-p_{i}^{j}\right) \cdot x_{i}^{j}\right)\right\} \\
B_{\varepsilon}(X)= & \exp \left\{-\mathrm{i} \sum_{j=1}^{M} \sum_{i=1}^{n} \mathcal{B}_{\varepsilon}^{i}\left(x_{i}^{j}\right)+\mathrm{i} \sum_{j=M+1}^{M+N} \sum_{i=1}^{n} \mathcal{B}_{\varepsilon}^{i}\left(x_{i}^{j}\right)\right\}:=\mathrm{e}^{-\mathrm{i} \mathfrak{B}_{\varepsilon}(X)} \\
\widehat{\Phi}_{0}(P)= & \prod_{i=1}^{M} \prod_{j=M+1}^{M+N} \widehat{\zeta}_{0}^{\varepsilon}\left(p_{1}^{i}\right)\left(\zeta_{0}^{\varepsilon}\right)^{*}\left(p_{1}^{j}\right) .
\end{aligned}
$$

Above, we also used the notation $p_{n+1}^{j}=k$ for all $j=1, \ldots, M+N$. We have

$$
\operatorname{Var}\left(\mathfrak{B}_{\varepsilon}(X)\right)=v_{\varepsilon}^{I_{M}, I_{M}}(X)+v_{\varepsilon}^{I_{N}, I_{N}}(X)-2 v_{\varepsilon}^{I_{M}, I_{N}}(X),
$$

where $I_{M}=\{1, \ldots, M\}, I_{N}=\{M+1, \ldots, M+N\}$ and

$$
v_{\varepsilon}^{A, B}(X):=\sum_{j_{a} \in A} \sum_{j_{b} \in B} \sum_{j, l=1}^{n} \int_{(j-1) h}^{j h} \int_{(l-1) h}^{l h} R_{\varepsilon}\left(u_{1}-u_{2}, \varepsilon^{\frac{s+\gamma}{2 \beta}-s}\left(x_{j}-x_{i}\right)\right) \mathrm{d} u_{1} \mathrm{~d} u_{2} .
$$

Case $\beta<\frac{1}{2}$ or $\gamma>\mathbf{0}$. It is not difficult to see that

$$
v_{\varepsilon}(X):=\operatorname{Var}\left(\mathfrak{B}_{\varepsilon, h}(X)\right) \rightarrow(M-N)^{2} v_{0},
$$

where $v_{0}$ is defined in (3.2). This, together with the change of variables

$$
p_{i}^{j} \rightarrow \varepsilon^{s-s_{c}} p_{i}^{j}+p_{i+1}^{j}, \quad \text { so that } \quad p_{i}^{j} \rightarrow \varepsilon^{s-s_{c}} \sum_{l=i}^{n} p_{l}^{j}+k:=\varepsilon^{s-s_{c}} \tilde{p}_{i}^{j}+k, \quad i=1, \ldots, n
$$


implies

$$
\begin{aligned}
\mathbb{E}\left\{\left(\zeta_{n}^{\varepsilon}(k)\right)^{M}\left(\zeta_{n}^{\varepsilon}(k)^{*}\right)^{N}\right\}= & \frac{1}{(2 \pi)^{(M+N) n d}} \int \mathrm{d} X \mathrm{~d} P \mathrm{e}^{-\frac{1}{2} v_{\varepsilon}(X)} \exp \left\{-\frac{\mathrm{i} \varepsilon^{s} h}{2}\left(\sum_{j=1}^{M} \sum_{i=2}^{n}\left|\tilde{p}_{i}^{j}\right|^{2}-\sum_{j=M+1}^{M+N} \sum_{i=2}^{n}\left|\tilde{p}_{i}^{j}\right|^{2}\right)\right\} \\
& \times \exp \left\{-\mathrm{i} \varepsilon^{s^{c}} h k \cdot\left(\sum_{j=1}^{M} \sum_{i=2}^{n} \tilde{p}_{i}^{j}-\sum_{j=M+1}^{M+N} \sum_{i=2}^{n} \tilde{p}_{i}^{j}\right)\right\} \\
& \times \exp \left\{\mathrm{i}\left(\sum_{j=1}^{M} \sum_{i=1}^{n} p_{i}^{j} \cdot x_{i}^{j}-\sum_{j=M+1}^{M+N} \sum_{i=1}^{n} p_{i}^{j} \cdot x_{i}^{j}\right)\right\} \widehat{\Phi}_{0}(P),
\end{aligned}
$$

so that, using (3.2), it comes

$$
\begin{aligned}
\lim _{\varepsilon \rightarrow 0} \mathbb{E}\left\{\left(\zeta_{n}^{\varepsilon}(k)\right)^{M}\left(\zeta_{n}^{\varepsilon}(k)^{*}\right)^{N}\right\}= & \frac{1}{(2 \pi)^{(M+N) n d}} \mathrm{e}^{-\frac{1}{2}(M-N)^{2} v_{0}} \int \mathrm{d} X \mathrm{~d} P \widehat{\phi_{0}}\left(\delta\left(s-s_{c}\right) \tilde{p}_{1}^{i}+k\right)^{M} \widehat{\phi}_{0}^{*}\left(\delta\left(s-s_{c}\right) \tilde{p}_{1}^{j}+k\right)^{N} \\
& \times \exp \left\{-\mathrm{i} \delta\left(s_{c}\right) h k \cdot\left(\sum_{j=1}^{M} \sum_{i=1}^{n-1} \tilde{p}_{i}^{j}-\sum_{j=M+1}^{M+N} \sum_{i=1}^{n-1} \tilde{p}_{i}^{j}\right)\right\} \\
& \times \exp \left\{\mathrm{i}\left(\sum_{j=1}^{M} \sum_{i=1}^{n} p_{i}^{j} \cdot x_{i}^{j}-\sum_{j=M+1}^{M+N} \sum_{i=1}^{n} p_{i}^{j} \cdot x_{i}^{j}\right)\right\} \\
= & \mathrm{e}^{-\frac{1}{2}(M-N)^{2} v_{0}} \int \mathrm{d} P \widehat{\phi}_{0}\left(\delta\left(s-s_{c}\right) \tilde{p}_{1}^{i}+k\right)^{M} \widehat{\phi}_{0}^{*}\left(\delta\left(s-s_{c}\right) \tilde{p}_{1}^{j}+k\right)^{N} \\
& \times \exp \left\{-\mathrm{i} \delta\left(s_{c}\right) h k \cdot\left(\sum_{j=1}^{M} \sum_{i=1}^{n-1} \tilde{p}_{i}^{j}-\sum_{j=M+1}^{M+N} \sum_{i=1}^{n-1} \tilde{p}_{i}^{j}\right)\right\} \prod_{i=1}^{n} \prod_{j=1}^{M+N} \delta\left(p_{i}^{j}\right) \\
= & \mathrm{e}^{-\frac{1}{2}(M-N)^{2} v_{0} \widehat{\phi}_{0}(k)^{M} \widehat{\phi}_{0}^{*}(k)^{N} .}
\end{aligned}
$$

Case $\boldsymbol{\beta}=\frac{1}{2}$ and $\gamma=\mathbf{0}$. The previous computation has to be replaced by

$$
\begin{aligned}
\lim _{\varepsilon \rightarrow 0} \mathbb{E}\left\{\left(\zeta_{n}^{\varepsilon}(k)\right)^{M}\left(\zeta_{n}^{\varepsilon}(k)^{*}\right)^{N}\right\}= & \int \mathrm{d} x \mathrm{~d} z_{1} \cdots \mathrm{d} z_{M+N} \mathrm{e}^{-\frac{1}{2}(M-N)^{2} v_{0}(X)} \prod_{j=1}^{M+N} \prod_{i=1}^{n-1} \delta\left(x_{i}^{j}-\delta\left(s_{c}\right) i k h-\delta\left(s-s_{c}\right) z^{j}\right) \\
& \times \mathrm{e}^{-\mathrm{i} k \cdot\left(\sum_{j=1}^{M+N} z^{j}\right)} \prod_{j=1}^{M} \phi_{0}\left(z^{j}\right) \prod_{j=M+1}^{M+N}\left(\phi_{0}\right)^{*}\left(z^{j}\right),
\end{aligned}
$$

where

$$
v_{0}(X)=v_{0}^{I_{M}, I_{M}}(X)+v_{0}^{I_{N}, I_{N}}(X)-2 v_{0}^{I_{M}, I_{N}}(X),
$$

with $I_{M}=\{1, \ldots, M\}, I_{N}=\{M+1, \ldots, M+N\}$ and

$$
v_{0}^{A, B}(X):=\sum_{j_{a} \in A} \sum_{j_{b} \in B} \sum_{j, l=1}^{n} \int_{(j-1) h}^{j h} \int_{(l-1) h}^{l h} R\left(u_{1}-u_{2},\left(x_{j}-x_{i}\right)\right) \mathrm{d} u_{1} \mathrm{~d} u_{2} .
$$


Specializing to $x_{i}^{j}=\delta\left(s_{c}\right) \mathrm{i} k h+\delta\left(s-s_{c}\right) z^{j}$ because of the Dirac measure, we find

$$
v_{0}(X)=(M-N)^{2} v_{0}(n, h),
$$

where $v_{0}(n, h)$ is defined in (3.4).

Since the moments of $\zeta_{n}^{\varepsilon}$ converge to the moments of $\zeta$, in order to show that $\zeta_{n}^{\varepsilon}$ converges in law to $\zeta$, one needs to show that the moments of $\zeta$ characterize uniquely its distribution. This is a consequence of Carleman's criterion. Indeed,

$$
\sum_{m=1}^{\infty}\left(\mathbb{E}\left\{|\zeta(k)|^{2 m}\right\}\right)^{-\frac{1}{2 m}} \geq\left|\widehat{\phi}_{0}(k)\right|^{-1} \sum_{m=1}^{\infty} 1=\infty
$$

so that we conclude that $\zeta_{n}^{\varepsilon}$ converges in law to $\zeta$.

\section{Proof of Theorem 2.2}

The first step of the proof is to derive the corresponding scheme for (2.16) from (1.5) and obtain a recursive expression. Then, thanks to the Markov inequality

$$
\mathbb{P}\left(\left|\left\langle W_{n, h}^{\varepsilon}-W(n h), \phi\right\rangle_{L^{2}\left(\mathbb{R}^{2 d}\right)}\right|^{2}>\eta\right) \leq \mathbb{E}\left(\left|\left\langle W_{n, h}^{\varepsilon}-W(n h), \phi\right\rangle_{L^{2}\left(\mathbb{R}^{2 d}\right)}\right|^{2}\right) / \eta,
$$

and the fact that $W_{n, h}^{\varepsilon}$ and $W(n h)$ are real-valued, it suffices to show the convergence of the first and second order moments of (2.16) applied to a test function $\phi \in L^{2}\left(\mathbb{R}^{2 d}\right)$ towards $\langle W(n h), \phi\rangle_{L^{2}\left(\mathbb{R}^{2 d}\right)}$ and $\langle W(n h), \phi\rangle_{L^{2}\left(\mathbb{R}^{2 d}\right)}^{2}$ respectively, where $W(t)$ is defined by (2.17). We have the following lemma, which offers an expression for $W_{n, h}^{\varepsilon}$ in the Fourier domain:

Lemma 4.1. The Fourier transform of $W_{n, h}^{\varepsilon}$ satisfies the expression

$$
\begin{aligned}
\widehat{W}_{n, h}^{\varepsilon}(q, y)= & \frac{1}{(2 \pi)^{d}} \int \mathrm{d} q_{1} \mathrm{~d} x_{1} \mathrm{e}^{-\mathrm{i}\left(q-q_{1}\right) \cdot x_{1}} \mathrm{e}^{-\mathrm{i}\left(\mathcal{B}_{n, h}^{\varepsilon}\left(x_{1}-\varepsilon^{s-s_{c}}\left(y+\varepsilon^{s_{c}} h q\right) / 2\right)-\mathcal{B}_{n, h}^{\varepsilon}\left(x_{1}+\varepsilon^{s-s_{c}}\left(y+\varepsilon^{s_{c}} h q\right) / 2\right)\right)} \\
& \times \widehat{W}_{n-1, h}^{\varepsilon}\left(q_{1}, y+\varepsilon^{s_{c}} h q\right)
\end{aligned}
$$

so that

$$
\begin{aligned}
\widehat{W}_{n, h}^{\varepsilon}(q, y)= & \frac{1}{(2 \pi)^{n d}} \int \mathrm{d} Q \mathrm{~d} x \mathrm{e}^{-\mathrm{i} \sum_{j=1}^{n}\left(q_{j-1}-q_{j}\right) \cdot x_{j}} \\
& \times \mathrm{e}^{-\mathrm{i} \sum_{j=1}^{n} \mathcal{B}_{j, h}^{\varepsilon}\left(x_{n-j+1}-\varepsilon^{s-s_{c}}\left(y+\varepsilon^{s_{c}} h \sum_{l=1}^{n-j+1} q_{l-1}\right) / 2\right)-\mathcal{B}_{j, h}^{\varepsilon}\left(x_{n-j+1}+\varepsilon^{s-s_{c}}\left(y+\varepsilon^{s_{c}} h \sum_{l=1}^{n-j+1} q_{l-1}\right) / 2\right)} \\
& \times \widehat{W}_{0}^{\varepsilon}\left(q_{n}, y+\varepsilon^{s_{c}} h \sum_{l=1}^{n} q_{l-1}\right),
\end{aligned}
$$

where $\mathcal{B}_{n, h}^{\varepsilon}$ is defined by (1.4), $X=\left(x_{1}, \ldots, x_{n}\right), Q=\left(q_{1}, \ldots, q_{n}\right)$, and $q_{0}=q$.

As a result, since for a zero-mean Gaussian variable $G$ with variance $\sigma^{2}$, we have $\mathbb{E}\left[\mathrm{e}^{\mathrm{i} G}\right]=\mathrm{e}^{-\sigma^{2} / 2}$, we just need to compute

$$
B_{n, h}^{1, \varepsilon}=\mathbb{E}\left[\left(\sum_{j=1}^{n} \mathcal{B}_{j, h}^{\varepsilon}\left(x_{n-j+1}-\varepsilon^{s-s_{c}}\left(y+\varepsilon^{s_{c}} h Q_{n, j}\right) / 2\right)-\mathcal{B}_{j, h}^{\varepsilon}\left(x_{n-j+1}+\varepsilon^{s-s_{c}}\left(y+\varepsilon^{s_{c}} h Q_{n, j}\right) / 2\right)\right)^{2}\right],
$$


and

$$
\begin{aligned}
B_{n, h}^{2, \varepsilon}= & \mathbb{E}\left[\left(\sum_{j=1}^{n} \mathcal{B}_{j, h}^{\varepsilon}\left(x_{n-j+1}^{1}-\varepsilon^{s-s_{c}}\left(y^{1}+\varepsilon^{s_{c}} h Q_{n, j}^{1}\right) / 2\right)-\mathcal{B}_{j, h}^{\varepsilon}\left(x_{n-j+1}^{1}+\varepsilon^{s-s_{c}}\left(y^{1}+\varepsilon^{s_{c}} h Q_{n, j}^{1}\right) / 2\right)\right.\right. \\
& \left.\left.+\mathcal{B}_{j, h}^{\varepsilon}\left(x_{n-j+1}^{2}-\varepsilon^{s-s_{c}}\left(y^{2}+\varepsilon^{s_{c}} h Q_{n, j}^{2}\right) / 2\right)-\mathcal{B}_{j, h}^{\varepsilon}\left(x_{n-j+1}^{2}+\varepsilon^{s-s_{c}}\left(y^{2}+\varepsilon^{s_{c}} h Q_{n, j}^{2}\right) / 2\right)\right)^{2}\right],
\end{aligned}
$$

to study the convergence of respectively the first and second moment of $\widehat{W}_{n, h}^{\varepsilon}$, where

$$
Q_{n, j}=\sum_{l=1}^{n-j+1} q_{l-1}
$$

It is the object of the following lemma:

Lemma 4.2. We have

$$
\lim _{\varepsilon \rightarrow 0} B_{n, h}^{1, \varepsilon}=2 n h \sigma(\theta)|y|^{\theta}, \quad \text { and } \quad \lim _{\varepsilon \rightarrow 0} B_{n, h}^{2, \varepsilon}=2 n h \sigma(\theta)\left(\left|y^{1}\right|^{\theta}+\left|y^{2}\right|^{\theta}\right)
$$

where $B_{n, h}^{1, \varepsilon}$ and $B_{n, h}^{2, \varepsilon}$ are respectively defined by (4.3) and (4.4).

We have finally the following result which concludes the proof of Theorem 2.2:

Lemma 4.3. We have for all $\phi \in L^{2}\left(\mathbb{R}^{2 d}\right)$,

$$
\lim _{\varepsilon \rightarrow 0} \mathbb{E}\left[\left\langle W_{n, h}^{\varepsilon}, \phi\right\rangle_{L^{2}\left(\mathbb{R}^{2 d}\right)}\right]=\frac{1}{(2 \pi)^{2 d}} \lim _{\varepsilon \rightarrow 0} \mathbb{E}\left[\left\langle\widehat{W}_{n, h}^{\varepsilon}, \widehat{\phi}\right\rangle_{L^{2}\left(\mathbb{R}^{2 d}\right)}\right]=\frac{1}{(2 \pi)^{2 d}}\langle\widehat{W}(n h), \widehat{\phi}\rangle_{L^{2}\left(\mathbb{R}^{2 d}\right)}=\langle W(n h), \phi\rangle_{L^{2}\left(\mathbb{R}^{2 d}\right)},
$$

and

$$
\lim _{\varepsilon \rightarrow 0} \mathbb{E}\left[\left\langle W_{n, h}^{\varepsilon}, \phi\right\rangle_{L^{2}\left(\mathbb{R}^{2 d}\right)}^{2}\right]=\frac{1}{(2 \pi)^{2 d}} \lim _{\varepsilon \rightarrow 0} \mathbb{E}\left[\left\langle\widehat{W}_{n, h}^{\varepsilon}, \widehat{\phi}\right\rangle_{L^{2}\left(\mathbb{R}^{2 d}\right)}^{2}\right]=\frac{1}{(2 \pi)^{2 d}}\langle\widehat{W}(n h), \widehat{\phi}\rangle_{L^{2}\left(\mathbb{R}^{2 d}\right)}^{2}=\langle W(n h), \phi\rangle_{L^{2}\left(\mathbb{R}^{2 d}\right)}^{2},
$$

where $W$ is defined by $(2.17)$.

The remaining part of this section consists in proving the three previous lemmas. 
Proof of Lemma 4.1. According to the definition of the Wigner transform (2.16) and (1.5):

$$
\begin{aligned}
\widehat{W}_{n, h}^{\varepsilon}(q, y)= & \int \mathrm{d} x \nu(\mathrm{d} \zeta) \mathrm{e}^{-\mathrm{i} q \cdot x} \Psi_{n}^{\varepsilon}\left(x-\varepsilon^{s-s_{c}} \frac{y}{2}, \zeta\right) \overline{\Psi_{n}^{\varepsilon}\left(x+\varepsilon^{s-s_{c}} \frac{y}{2}, \zeta\right)} \\
= & \frac{1}{(2 \pi)^{2 d}} \int \mathrm{d} x \mathrm{~d} k_{1} \mathrm{~d} x_{1} \mathrm{~d} \tilde{k}_{1} \mathrm{~d} \tilde{x}_{1} \nu(\mathrm{d} \zeta) \mathrm{e}^{-\mathrm{i} q \cdot x} \mathrm{e}^{\mathrm{i} k_{1} \cdot\left(x-\varepsilon^{s-s_{c}} y / 2-x_{1}\right)} \mathrm{e}^{-\mathrm{i} \tilde{k}_{1} \cdot\left(x+\varepsilon^{s-s_{c}} y / 2-\tilde{x}_{1}\right)} \\
& \times \mathrm{e}^{-\mathrm{i} \varepsilon^{s} h\left|k_{1}\right|^{2} / 2} \mathrm{e}^{\mathrm{i} \varepsilon^{s} h\left|\tilde{k}_{1}\right|^{2} / 2} \mathrm{e}^{-\mathrm{i}\left(\mathcal{B}_{n, h}^{\varepsilon}\left(x_{1}\right)-\mathcal{B}_{n, h}^{\varepsilon}\left(\tilde{x}_{1}\right)\right)} \Psi_{n-1}^{\varepsilon}\left(x_{1}, \zeta\right) \overline{\Psi_{n-1}^{\varepsilon}\left(\tilde{x}_{1}, \zeta\right)} \\
= & \frac{1}{(2 \pi)^{d}} \int \mathrm{d} k_{1} \mathrm{~d} x_{1} \mathrm{~d} \tilde{k}_{1} \mathrm{~d} \tilde{x}_{1} \nu(\mathrm{d} \zeta) \delta\left(k_{1}-q-\tilde{k}_{1}\right) \mathrm{e}^{-\mathrm{i} k_{1} \cdot\left(\varepsilon^{s-s_{c}} y / 2+x_{1}\right)} \mathrm{e}^{-\mathrm{i} \tilde{k}_{1} \cdot\left(\varepsilon^{s-s_{c}} y / 2-\tilde{x}_{1}\right)} \\
& \times \mathrm{e}^{-\mathrm{i} \varepsilon^{s} h\left|k_{1}\right|^{2} / 2} \mathrm{e}^{\mathrm{i} \varepsilon^{s} h\left|\tilde{k}_{1}\right|^{2} / 2} \mathrm{e}^{-\mathrm{i}\left(\mathcal{B}_{n, h}^{\varepsilon}\left(x_{1}\right)-\mathcal{B}_{n, h}^{\varepsilon}\left(\tilde{x}_{1}\right)\right)} \Psi_{n-1}^{\varepsilon}\left(x_{1}, \zeta\right) \overline{\Psi_{n-1}^{\varepsilon}\left(\tilde{x}_{1}, \zeta\right)} \\
= & \frac{1}{(2 \pi)^{d}} \int \mathrm{d} x_{1} \mathrm{~d} \tilde{k}_{1} \mathrm{~d} \tilde{x}_{1} \nu(\mathrm{d} \zeta) \mathrm{e}^{\mathrm{i} \tilde{k}_{1} \cdot\left(\tilde{x}_{1}-x_{1}-\varepsilon^{s-s_{c}} y-\varepsilon^{s} h q\right)} \mathrm{e}^{-\mathrm{i} q \cdot\left(\varepsilon^{s-s_{c}} y / 2+x_{1}\right)} \\
& \times \mathrm{e}^{-\mathrm{i} \varepsilon^{s} h|q|^{2} / 2} \mathrm{e}^{-\mathrm{i}\left(\mathcal{B}_{n, h}^{\varepsilon}\left(x_{1}\right)-\mathcal{B}_{n, h}^{\varepsilon}\left(\tilde{x}_{1}\right)\right)} \Psi_{n-1}^{\varepsilon}\left(x_{1}, \zeta\right) \overline{\Psi_{n-1}^{\varepsilon}\left(\tilde{x}_{1}, \zeta\right)} \\
= & \int \mathrm{d} x_{1} \mathrm{~d} \tilde{x}_{1} \nu(\mathrm{d} \zeta) \delta\left(\tilde{x}_{1}-x_{1}-\varepsilon^{s-s_{c}} y-\varepsilon^{s} h q\right) \mathrm{e}^{-\mathrm{i} q \cdot\left(\varepsilon^{s-s_{c}} y / 2+x_{1}\right)} \\
& \times \mathrm{e}^{-\mathrm{i} \varepsilon^{s} h|q|^{2} / 2} \mathrm{e}^{-\mathrm{i}\left(\mathcal{B}_{n, h}^{\varepsilon}\left(x_{1}\right)-\mathcal{B}_{n, h}^{\varepsilon}\left(\tilde{x}_{1}\right)\right)} \Psi_{n-1}^{\varepsilon}\left(x_{1}, \zeta\right) \overline{\Psi_{n-1}^{\varepsilon}\left(\tilde{x}_{1}, \zeta\right)} \\
= & \int \mathrm{d} x_{1} \nu(\mathrm{d} \zeta) \mathrm{e}^{-\mathrm{i} q \cdot\left(\varepsilon^{s-s_{c}} y / 2+x_{1}\right)} \mathrm{e}^{-\mathrm{i} \varepsilon^{s} h|q|^{2} / 2} \mathrm{e}^{-\mathrm{i}\left(\mathcal{B}_{n, h}^{\varepsilon}\left(x_{1}\right)-\mathcal{B}_{n, h}^{\varepsilon}\left(x_{1}+\varepsilon^{s-s_{c}} y+\varepsilon^{s} h q\right)\right)} \\
& \times \Psi_{n-1}^{\varepsilon}\left(x_{1}, \zeta\right) \overline{\Psi_{n-1}^{\varepsilon}\left(x_{1}+\varepsilon^{s-s_{c}} y+\varepsilon^{s} h q, \zeta\right)}
\end{aligned}
$$

Then, thanks to the change of coordinates $x_{1} \rightarrow x_{1}-\varepsilon^{s-s_{c}} y / 2-\varepsilon^{s} h q / 2$, we have

$$
\begin{aligned}
\widehat{W}_{n, h}^{\varepsilon}(q, y)= & \int \mathrm{d} x_{1} \nu(\mathrm{d} \zeta) \mathrm{e}^{-\mathrm{i} q \cdot x_{1}} \mathrm{e}^{-\mathrm{i}\left(\mathcal{B}_{n, h}^{\varepsilon}\left(x_{1}-\varepsilon^{s-s_{c}} y / 2-\varepsilon^{s} h q / 2\right)-\mathcal{B}_{n, h}^{\varepsilon}\left(x_{1}+\varepsilon^{s-s_{c}} y / 2+\varepsilon^{s} h q / 2\right)\right)} \\
& \times \Psi_{n-1}^{\varepsilon}\left(x_{1}-\varepsilon^{s-s_{c}} y / 2-\varepsilon^{s} h q / 2, \zeta\right) \overline{\Psi_{n-1}^{\varepsilon}\left(x_{1}+\varepsilon^{s-s_{c}} y / 2+\varepsilon^{s} h q / 2, \zeta\right)} \\
= & \int \mathrm{d} x_{1} \mathrm{e}^{-\mathrm{i} q \cdot x_{1}} \mathrm{e}^{-\mathrm{i}\left(\mathcal{B}_{n, h}^{\varepsilon}\left(x_{1}-\varepsilon^{s-s_{c}} y / 2-\varepsilon^{s} h q / 2\right)-\mathcal{B}_{n, h}^{\varepsilon}\left(x_{1}+\varepsilon^{s-s_{c}} y / 2+\varepsilon^{s} h q / 2\right)\right)} \\
& \times \frac{1}{(2 \pi)^{d}} \int \mathrm{d} q_{1} \mathrm{e}^{\mathrm{i} q_{1} \cdot x_{1}} \widehat{W}_{n-1, h}^{\varepsilon}\left(q_{1}, y+\varepsilon^{s_{c}} h q\right),
\end{aligned}
$$

by inverse Fourier transform, which proves (4.1). Moreover, using recursively (4.1),

$$
\begin{aligned}
\widehat{W}_{n, h}^{\varepsilon}(q, y)= & \frac{1}{(2 \pi)^{n d}} \int \mathrm{d} Q \mathrm{~d} X \widehat{W}_{0}^{\varepsilon}\left(q_{n}, y+\varepsilon^{s_{c}} h \sum_{l=1}^{n} q_{l-1}\right) \mathrm{e}^{-\mathrm{i} \sum_{j=1}^{n}\left(q_{j-1}-q_{j}\right) \cdot x_{j}} \\
& \times \mathrm{e}^{-\mathrm{i} \sum_{j=1}^{n} \mathcal{B}_{n-j+1, h}^{\varepsilon}\left(x_{j}-\varepsilon^{s-s_{c}}\left(y+\varepsilon^{s_{c}} h \sum_{l=1}^{j} q_{l-1}\right) / 2\right)-\mathcal{B}_{n-j+1, h}^{\varepsilon}\left(x_{j}+\varepsilon^{s-s_{c}}\left(y+\varepsilon^{s_{c}} h \sum_{l=1}^{j} q_{l-1}\right) / 2\right)}
\end{aligned}
$$

one obtain (4.2) with the change of index $n-j+1 \rightarrow j$ at the second line, which concludes the proof of Lemma 4.1. 
Proof of Lemma 4.2. Let us begin with $B_{n, h}^{1, \varepsilon}$. Using (2.1)-(2.3), we have

$$
\begin{gathered}
B_{n, h}^{1, \varepsilon}=\frac{2 \varepsilon^{1-s}}{(2 \pi)^{d+1}} \sum_{j, l=1}^{n} \int \mathrm{d} \omega \mathrm{d} p \mathrm{e}^{\mathrm{i} \omega(j-l) h} \frac{a(p) \mu|p|^{2 \beta}}{|p|^{d+2(\alpha-1)}\left(\mu^{2}|p|^{4 \beta}+\omega^{2} \varepsilon^{2(s+\gamma)}\right)} \int_{0}^{h} \int_{0}^{h} \mathrm{~d} u_{1} \mathrm{~d} u_{2} \mathrm{e}^{\mathrm{i} \omega\left(u_{1}-u_{2}\right)} \\
\times \mathrm{e}^{\mathrm{i} p \cdot\left(x_{n-j+1}-x_{n-l+1}\right) / \varepsilon^{s}}\left(\mathrm{e}^{\left.-\mathrm{i} h p \cdot\left(Q_{n, j}-Q_{n, l}\right) / 2\right)}+\mathrm{e}^{\left.\mathrm{i} h p \cdot\left(Q_{n, j}-Q_{n, l}\right) / 2\right)}\right. \\
\left.\quad-\mathrm{e}^{-\mathrm{i} p \cdot\left(y / \varepsilon^{s_{c}}+h\left(Q_{n, j}+Q_{n, l}\right) / 2\right)}-\mathrm{e}^{\mathrm{i} p \cdot\left(y / \varepsilon^{s_{c}}+h\left(Q_{n, j}+Q_{n, l}\right) / 2\right)}\right) .
\end{gathered}
$$

Then, after the change of variable $p \rightarrow \varepsilon^{s_{c}} p$, and (2.13), we have

$$
\begin{aligned}
B_{n, h}^{1, \varepsilon}= & \frac{2}{(2 \pi)^{d+1}} \sum_{j, l=1}^{n} \int \mathrm{d} \omega \mathrm{d} p \mathrm{e}^{\mathrm{i} \omega(j-l) h} \frac{a\left(\varepsilon^{s_{c}} p\right) \mu|p|^{2 \beta}}{|p|^{d+2(\alpha-1)}\left(\mu^{2}|p|^{4 \beta}+\omega^{2} \varepsilon^{2\left(s+\gamma-2 \beta s_{c}\right)}\right)} \int_{0}^{h} \int_{0}^{h} \mathrm{~d} u_{1} \mathrm{~d} u_{2} \mathrm{e}^{\mathrm{i} \omega\left(u_{1}-u_{2}\right)} \\
& \times \mathrm{e}^{\mathrm{i} p \cdot\left(x_{n-j+1}-x_{n-l+1}\right) / \varepsilon^{s-s_{c}}}\left(\mathrm{e}^{\left.-\mathrm{i} \varepsilon^{s_{c}} h p \cdot\left(Q_{n, j}-Q_{n, l}\right) / 2\right)}+\mathrm{e}^{\left.\mathrm{i} \varepsilon^{\varepsilon_{c}} h p \cdot\left(Q_{n, j}-Q_{n, l}\right) / 2\right)}\right. \\
& \left.-\mathrm{e}^{-\mathrm{i} p \cdot\left(y+\varepsilon^{s c} h\left(Q_{n, j}+Q_{n, l}\right) / 2\right)}-\mathrm{e}^{\mathrm{i} p \cdot\left(y+\varepsilon^{\varepsilon^{c}} h\left(Q_{n, j}+Q_{n, l}\right) / 2\right)}\right) .
\end{aligned}
$$

Moreover, $s+\gamma-2 \beta s_{c}>0$ since $s>1 /\left(2 \kappa_{\gamma}\right)$, so that the diagonal terms $(j=l)$ in the previous sum gives when $\varepsilon \rightarrow 0$

$$
\frac{4}{(2 \pi)^{d}} \sum_{j=1}^{n} \int_{0}^{h} \int_{0}^{h} \mathrm{~d} u_{1} \mathrm{~d} u_{2} \delta\left(u_{1}-u_{2}\right) \int \mathrm{d} p \frac{a(0)}{\mu|p|^{d+\theta}}\left(1-\mathrm{e}^{-\mathrm{i} p \cdot y}\right)=2 n h \sigma(\theta)|y|^{\theta} .
$$

Finally, the off diagonal terms $(j \neq l)$ are negligible as $\varepsilon \rightarrow 0$ since $s_{c}<s$ so that the fast phase $\mathrm{e}^{\mathrm{i} p \cdot\left(x_{n-j+1}-x_{n-l+1}\right) / \varepsilon^{s-s_{c}}}$ cannot be cancelled. Now to compute $\lim _{\varepsilon \rightarrow 0} B_{n, h}^{2, \varepsilon}$ we follow what we have just done for $B_{n, h}^{1, \varepsilon}$. Using (2.1)-(2.3), the change of variable $p \rightarrow \varepsilon^{s_{c}} p$, and (2.13), we have

$$
\begin{aligned}
B_{n, h}^{2, \varepsilon}= & \frac{2}{(2 \pi)^{d+1}} \sum_{j, l=1}^{n} \int \mathrm{d} \omega \mathrm{d} p \mathrm{e}^{\mathrm{i} \omega(j-l) h} \int_{0}^{h} \int_{0}^{h} \mathrm{~d} u_{1} \mathrm{~d} u_{2} \mathrm{e}^{\mathrm{i} \omega\left(u_{1}-u_{2}\right)} \frac{a(p) \mu|p|^{2 \beta}}{|p|^{d+2(\alpha-1)}\left(\mu^{2}|p|^{4 \beta}+\omega^{2} \varepsilon^{2\left(s+\gamma-2 \beta s_{c}\right)}\right)} \\
& \times\left[\sum _ { r _ { 1 } , r _ { 2 } = 1 } ^ { 2 } \mathrm { e } ^ { \mathrm { i } p ( x _ { n - j + 1 } ^ { r _ { 1 } } - x _ { n - l + 1 } ^ { r _ { 2 } } ) / \varepsilon ^ { s - s _ { c } } } \left(\mathrm{e}^{\left.-\mathrm{i} p \cdot\left(y^{r_{1}}-y^{r_{2}}+h \varepsilon^{s_{c}}\left(Q_{n, j}^{r_{1}}-Q_{n, l}^{r_{2}}\right)\right) / 2\right)}-\mathrm{e}^{\left.-\mathrm{i} p \cdot\left(y^{r_{1}}+y^{r_{2}}+h \varepsilon^{s_{c}}\left(Q_{n, j}^{r_{1}}+Q_{n, l}^{r_{2}}\right)\right) / 2\right)}\right.\right. \\
& \left.\left.-\mathrm{e}^{\left.\mathrm{i} p \cdot\left(y^{r_{1}}+y^{r_{2}}+h \varepsilon^{s_{c}}\left(Q_{n, j}^{r_{1}}+Q_{n, l}^{r_{2}}\right)\right) / 2\right)}+\mathrm{e}^{\left.\mathrm{i} p \cdot\left(y^{r_{1}}-y^{r_{2}}+h \varepsilon^{s_{c}}\left(Q_{n, j}^{r_{1}}-Q_{n, l}^{r_{2}}\right)\right) / 2\right)}\right)\right]
\end{aligned}
$$

Moreover, since $s>1 /\left(2 \kappa_{\gamma}\right)$, we have $s+\gamma-2 \beta s_{c}>0$ so that the diagonal terms $\left(j=l\right.$ and $\left.r_{1}=r_{2}\right)$ in the previous sum gives when $\varepsilon \rightarrow 0$

$$
\frac{4}{(2 \pi)^{d}} \sum_{j=1}^{n} \int_{0}^{h} \int_{0}^{h} \mathrm{~d} u_{1} \mathrm{~d} u_{2} \delta\left(u_{1}-u_{2}\right) \int \mathrm{d} p \frac{a(0)}{\mu|p|^{d+\theta}}\left(1-\mathrm{e}^{-\mathrm{i} p \cdot y^{1}}+1-\mathrm{e}^{\mathrm{i} p \cdot y^{2}}\right)=2 n h \sigma(\theta)\left(\left|y^{1}\right|^{\theta}+\left|y^{2}\right|^{\theta}\right) .
$$

The terms corresponding to the fast phases $\mathrm{e}^{\mathrm{i} p \cdot\left(x_{n-j+1}^{1}-x_{n-j+1}^{2}\right) / \varepsilon^{s-s_{c}}}$, which cannot be cancelled, give rise to negligible terms as $\varepsilon \rightarrow 0$. Finally, all the terms of the off diagonal $\left(j \neq l\right.$ or $\left.r_{1} \neq r_{2}\right)$ are also negligible for exactly the same reason, that conclude the proof of Lemma 4.2. 
Proof of Lemma 4.3. Applying Lemma 4.2 to the expectation of (4.2), we have

$$
\begin{aligned}
\lim _{\varepsilon \rightarrow 0} \mathbb{E}\left[\left\langle\widehat{W}_{n, h}^{\varepsilon}, \widehat{\phi}\right\rangle_{L^{2}\left(\mathbb{R}^{2 d}\right)}\right] & =\frac{1}{(2 \pi)^{n d}} \int \mathrm{d} Q \mathrm{~d} X \mathrm{~d} q \mathrm{~d} y \mathrm{e}^{-\mathrm{i} \sum_{j=1}^{n}\left(q_{j-1}-q_{j}\right) \cdot x_{j}} \mathrm{e}^{-n h \sigma(\theta)|y|^{\theta}} \widehat{W}_{0}\left(q_{n}, y\right) \overline{\widehat{\phi}(q, y)} \\
& =\int \mathrm{d} Q \mathrm{~d} q \mathrm{~d} y \prod_{j=1}^{n} \delta\left(q_{j-1}-q_{j}\right) \mathrm{e}^{-n h \sigma(\theta)|y|^{\theta} \widehat{W}_{0}}\left(q_{n}, y\right) \overline{\widehat{\phi}(q, y)} \\
& =\int \mathrm{d} q \mathrm{~d} y \mathrm{e}^{-n h \sigma(\theta)|y|^{\theta}} \widehat{W}_{0}(q, y) \overline{\widehat{\phi}(q, y)},
\end{aligned}
$$

so that $\lim _{\varepsilon \rightarrow 0} \mathbb{E}\left[\left\langle W_{n, h}^{\varepsilon}, \phi\right\rangle_{L^{2}\left(\mathbb{R}^{d}\right)}\right]=\langle W(n h), \phi\rangle_{L^{2}\left(\mathbb{R}^{d}\right)}$. Moreover,

$$
\begin{aligned}
\lim _{\varepsilon \rightarrow 0} \mathbb{E}\left[\left\langle\widehat{W}_{n, h}^{\varepsilon}, \widehat{\phi}\right\rangle_{L^{2}\left(\mathbb{R}^{2 d}\right)}\left\langle\widehat{W}_{n, h}^{\varepsilon}, \widehat{\phi}\right\rangle_{L^{2}}\left(\mathbb{R}^{2 d}\right)\right]=\frac{1}{(2 \pi)^{2 n d}} \int \mathrm{d} Q^{1} \mathrm{~d} X^{1} \mathrm{~d} Q^{2} \mathrm{~d} X^{2} \mathrm{~d} q_{1} \mathrm{~d} q_{2} \mathrm{~d} y_{1} \mathrm{~d} y_{2} \overline{\widehat{\phi}\left(q_{1}, y_{1}\right) \widehat{\phi}\left(q_{2}, y_{2}\right)} \\
\times \mathrm{e}^{-\mathrm{i} \sum_{j=1}^{n}\left(q_{j-1}^{1}-q_{j}^{1}\right) \cdot x_{j}^{1}} \mathrm{e}^{-\mathrm{i} \sum_{j=1}^{n}\left(q_{j-1}^{2}-q_{j}^{2}\right) \cdot x_{j}^{2}} \mathrm{e}^{-n h \sigma(\theta)\left(\left|y^{1}\right|^{\theta}+\left|y^{2}\right|^{\theta}\right)} \widehat{W}_{0}\left(q_{n}^{1}, y^{1}\right) \widehat{W}_{0}\left(q_{n}^{2}, y^{2}\right) \\
=\int \mathrm{d} Q^{1} \mathrm{~d} Q^{2} \prod_{j=1}^{n} \delta\left(q_{j-1}^{1}-q_{j}^{1}\right) \delta\left(q_{j-1}^{2}-q_{j}^{2}\right) \mathrm{e}^{-n h \sigma(\theta)\left(\left|y^{1}\right|^{\theta}+\left|y^{2}\right|^{\theta}\right)} \\
\times \widehat{W}_{0}\left(q_{n}^{1}, y^{1}\right) \widehat{W}_{0}\left(q_{n}^{2}, y^{2}\right) \overline{\widehat{\phi}\left(q_{1}, y_{1}\right) \widehat{\phi}\left(q_{2}, y_{2}\right)} \\
=\int \mathrm{d} q_{1} \mathrm{~d} q_{2} \mathrm{~d} y_{1} \mathrm{~d} y_{2} \mathrm{e}^{-n h \sigma(\theta)\left(\left|y^{1}\right|^{\theta}+\left|y^{2}\right|^{\theta}\right)} \widehat{W}_{0}\left(q^{1}, y^{1}\right) \widehat{W}_{0}\left(q^{2}, y^{2}\right) \overline{\hat{\phi}\left(q_{1}, y_{1}\right) \widehat{\phi}\left(q_{2}, y_{2}\right)}
\end{aligned}
$$

so that $\lim _{\varepsilon \rightarrow 0} \mathbb{E}\left[\left\langle W_{n, h}^{\varepsilon}, \phi\right\rangle_{L^{2}\left(\mathbb{R}^{2 d}\right)}^{2}\right]=\langle W(n h), \phi\rangle_{L^{2}\left(\mathbb{R}^{2 d}\right)}^{2}$, which concludes the proof of Lemma 4.3.

\section{Proof of Theorem 2.3}

The proof of is a generalization of the proof of Theorem 2.2, the main difference in Theorem 2.3 is that the limit of $W_{n, h}^{\varepsilon}$ is a random variable and not deterministic, and that the convergence holds strongly in $L^{2}\left(\mathbb{R}^{2 d}\right)$ since the $L^{2}\left(\mathbb{R}^{2 d}\right)$ norm of $W_{n, h}^{\varepsilon}$ is conserved at the limit. Once the convergence in distribution of $W_{n, h}^{\varepsilon}$ to $W(n h)$ on $L^{2}\left(\mathbb{R}^{2 d}\right)$ equipped with the weak topology is proved, one can use the Skorohod's representation theorem [5], Theorem 6.7 to construct respectively $\tilde{W}_{n, h}^{\varepsilon}$ and $\tilde{W}_{n, h}$ having the same distributions as $W_{n, h}^{\varepsilon}$ and $W(n h)$, and such that $\tilde{W}_{n, h}^{\varepsilon}$ converges almost surely in $L^{2}\left(\mathbb{R}^{2 d}\right)$ equipped with the weak topology. However, since $\left\|W_{n, h}^{\varepsilon}\right\|_{L^{2}\left(\mathbb{R}^{2 d}\right)}=\|W(n h)\|_{L^{2}\left(\mathbb{R}^{2 d}\right)}=\left\|W_{0}\right\|_{L^{2}\left(\mathbb{R}^{2 d}\right)}$, we also have $\left\|\tilde{W}_{n, h}^{\varepsilon}\right\|_{L^{2}\left(\mathbb{R}^{2 d}\right)}=\left\|\tilde{W}_{n, h}\right\|_{L^{2}\left(\mathbb{R}^{2 d}\right)}=\left\|W_{0}\right\|_{L^{2}\left(\mathbb{R}^{2 d}\right)}$ almost surely. As a result, since weak convergence together with convergence of the norms imply strong convergence, we can conclude that $\tilde{W}_{n, h}^{\varepsilon}$ converges almost surely, and then in distribution, in $L^{2}\left(\mathbb{R}^{2 d}\right)$ equipped with the strong topology.

More precisely, since $\left(W_{n, h}^{\varepsilon}\right)$ is a bounded random variable (in $L^{2}\left(\mathbb{R}^{2 d}\right)$ ), we only need to show the convergence of the moments to obtain convergence in distribution on $L^{2}\left(\mathbb{R}^{2 d}\right)$ equipped with the weak topology, that is for all $N \in \mathbb{N},\left(m_{1}, \ldots, m_{N}\right) \in \mathbb{N}^{N},\left(\phi_{1}, \ldots, \phi_{N}\right) \in L^{2}\left(\mathbb{R}^{2 N d}\right)$,

$$
\lim _{\varepsilon \rightarrow 0} \mathbb{E}\left[\prod_{r=1}^{N}\left\langle W_{n, h}^{\varepsilon}, \phi_{r}\right\rangle_{L^{2}\left(\mathbb{R}^{2 d}\right)}^{m_{r}}\right]=\mathbb{E}\left[\prod_{r=1}^{N}\left\langle W(n h), \phi_{r}\right\rangle_{L^{2}\left(\mathbb{R}^{2 d}\right)}^{m_{r}}\right],
$$

where $W(n h)$ is now defined by (2.19). For this, and according to (4.2), we will need the following lemma:

Lemma 5.1. Let us introduce

$$
B_{n, h}^{3, \varepsilon}=\mathbb{E}\left[\left(\sum_{r=1}^{N} \sum_{l=1}^{m_{r}} \sum_{j=1}^{n} \mathcal{B}_{j, h}^{\varepsilon}\left(x_{n-j+1}^{r, l}-\varepsilon^{s-s_{c}}\left(y^{r, l}+\varepsilon^{s_{c}} h Q_{n, j}^{r, l}\right) / 2\right)-\mathcal{B}_{j, h}^{\varepsilon}\left(x_{n-j+1}^{r, l}+\varepsilon^{s-s_{c}}\left(y^{r, l}+\varepsilon^{s_{c}} h Q_{n, j}^{r, l}\right) / 2\right)\right)^{2}\right]
$$


Then, we have

$$
\begin{aligned}
\lim _{\varepsilon \rightarrow 0} B_{n, h}^{3, \varepsilon}= & \frac{2 h}{(2 \pi)^{d}} \int \mathrm{d} p \frac{a(0)}{\mu|p|^{d+\theta}}\left[\sum_{j=1}^{n} \sum_{r_{1}, r_{2}=1}^{N} \sum_{l_{1}, l_{2}=1}^{m_{r}} \mathrm{e}^{\mathrm{i} p \cdot\left(x_{n-j+1}^{r_{1}, l_{1}}-x_{n-j+1}^{r_{2}, l_{2}}\right)}\right. \\
& \left.\times\left(\mathrm{e}^{-\mathrm{i} p \cdot\left(y^{r_{1}, l_{1}}-y^{r_{2}, l_{2}}\right) / 2}-\mathrm{e}^{-\mathrm{i} p \cdot\left(y^{r_{1}, l_{1}}+y^{r_{2}, l_{2}}\right) / 2}-\mathrm{e}^{\mathrm{i} p \cdot\left(y^{r_{1}, l_{1}}+y^{r_{2}, l_{2}}\right) / 2}+\mathrm{e}^{\mathrm{i} p \cdot\left(y^{r_{1}, l_{1}}-y^{r_{2}, l_{2}}\right) / 2}\right)\right] .
\end{aligned}
$$

Hence, using the previous lemma and (4.2) we obtain:

$$
\begin{aligned}
& \lim _{\varepsilon \rightarrow 0} \mathbb{E}\left[\prod_{r=1}^{N}\left\langle W_{n, h}^{\varepsilon}, \phi_{r}\right\rangle_{L^{2}\left(\mathbb{R}^{2 d}\right)}^{m_{r}}\right]=\lim _{\varepsilon \rightarrow 0} \mathbb{E}\left[\prod_{r=1}^{N} \frac{1}{(2 \pi)^{d}}\left\langle\widehat{W}_{n, h}^{\varepsilon}, \widehat{\phi}_{r}\right\rangle_{L^{2}\left(\mathbb{R}^{2 d}\right)}^{m_{r}}\right] \\
& =\frac{1}{(2 \pi)^{(n+1)\left(m_{1}+\cdots+m_{N}\right) d}} \int \mathrm{d} Q^{N} \mathrm{~d} X^{N} \mathrm{~d} y^{N} \prod_{r=1}^{N} \prod_{l=1}^{m_{r}} \mathrm{e}^{-\mathrm{i} \sum_{j=1}^{n}\left(q_{j-1}^{r, l}-q_{j}^{r, l}\right) \cdot x_{j}^{r, l}} \\
& \times \mathrm{e}^{-\lim _{\varepsilon \rightarrow 0} B_{n, h}^{3, \varepsilon} / 2} \prod_{r=1}^{N} \prod_{l=1}^{m_{r}} \widehat{W}_{0}\left(q_{n}^{r, l}, y^{r, l}\right) \overline{\widehat{\phi}_{r}\left(q^{r, l}, y^{r, l}\right)}, \\
& =\frac{1}{(2 \pi)^{3\left(m_{1}+\cdots+m_{N}\right) d}} \int \mathrm{d} q^{N} \mathrm{~d} q_{n}^{N} \mathrm{~d} X^{n} \mathrm{~d} y^{N} \prod_{r=1}^{N} \prod_{l=1}^{m_{r}} \mathrm{e}^{-\mathrm{i}\left(q^{r, l} \cdot x_{1}^{r, l}-q_{n}^{r, l} \cdot x_{n}^{r, l}\right)} \delta\left(x_{j+1}^{r, l}-x_{j}^{r, l}\right) \\
& \times \mathrm{e}^{-\lim _{\varepsilon \rightarrow 0} B_{n, h}^{3, \varepsilon} / 2} \prod_{r=1}^{N} \prod_{l=1}^{m_{r}} \widehat{W}_{0}\left(q_{n}^{r, l}, y^{r, l}\right) \overline{\widehat{\phi}_{r}\left(q^{r, l}, y^{r, l}\right)}, \\
& =\frac{1}{(2 \pi)^{\left(m_{1}+\cdots+m_{N}\right) d}} \int \mathrm{d} q^{N} \mathrm{~d} q_{n}^{N} \mathrm{~d} x_{1}^{N} \mathrm{~d} y^{N} \prod_{r=1}^{N} \prod_{l=1}^{m_{r}} \mathrm{e}^{\mathrm{i}\left(q^{r, l}-q_{n}^{r, l}\right) \cdot x_{1}} \widehat{W}_{0}\left(q_{n}^{r, l}, y^{r, l}\right) \widehat{\phi}_{r}\left(q^{r, l}, y^{r, l}\right) \\
& \times \exp \left(-\frac{n h}{(2 \pi)^{d}} \int \mathrm{d} p \frac{a(0)}{\mu|p|^{d+\theta}}\left[\sum_{r_{1}, r_{2}=1}^{N} \sum_{l_{1}, l_{2}=1}^{m_{r}} \mathrm{e}^{\mathrm{i} p \cdot\left(x_{1}^{r_{1}, l_{1}}-x_{1}^{r_{2}, l_{2}}\right)}\right.\right. \\
& \times\left(\mathrm{e}^{-\mathrm{i} p \cdot\left(y^{r_{1}, l_{1}}-y^{r_{2}, l_{2}}\right) / 2}-\mathrm{e}^{-\mathrm{i} p \cdot\left(y^{r_{1}, l_{1}}+y^{r_{2}, l_{2}}\right) / 2}\right. \\
& \left.\left.\left.-\mathrm{e}^{\mathrm{i} p \cdot\left(y^{r_{1}, l_{1}}+y^{r_{2}, l_{2}}\right) / 2}+\mathrm{e}^{\mathrm{i} p \cdot\left(y^{r_{1}, l_{1}}-y^{r_{2}, l_{2}}\right) / 2}\right)\right]\right) \\
& =\frac{1}{(2 \pi)^{3\left(m_{1}+\cdots+m_{N}\right) d}} \int \mathrm{d} q^{N} \mathrm{~d} q_{n}^{N} \mathrm{~d} x_{1}^{N} \prod_{r=1}^{N} \prod_{l=1}^{m_{r}} \mathrm{e}^{\mathrm{i}\left(q^{r, l}-q_{n}^{r, l}\right) \cdot x_{1}} \\
& \times \prod_{r=1}^{N} \prod_{l=1}^{m_{r}} \widehat{W}_{0}\left(q_{n}^{r, l}, y^{r, l}\right) \widehat{\phi}_{r}\left(q^{r, l}, y^{r, l}\right) \\
& \times \exp \left(-\mathbb{E}\left[\left(\sum_{r=1}^{N} \sum_{l=1}^{m_{r}} \int \mathcal{B}_{n h}(\mathrm{~d} p) \mathrm{e}^{\mathrm{i} p \cdot x_{1}^{r, l}}\left(\mathrm{e}^{-\mathrm{i} y^{r, l} \cdot p / 2}-\mathrm{e}^{\mathrm{i} y^{r, l} \cdot p / 2}\right)\right)^{2}\right] / 2\right)
\end{aligned}
$$

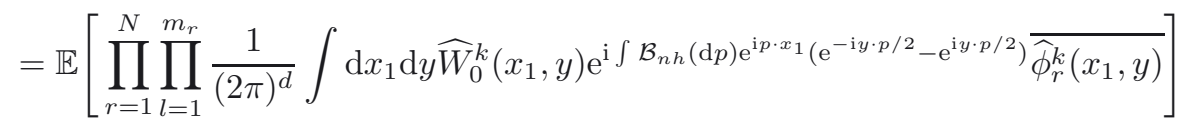

$$
\begin{aligned}
& =\mathbb{E}\left[\prod_{r=1}^{N} \prod_{l=1}^{m_{r}}\left\langle W(n h), \phi_{r}\right\rangle_{L^{2}\left(\mathbb{R}^{2 d}\right)}^{m_{r}}\right],
\end{aligned}
$$


where $W$ is defined in (2.19). This concludes the proof of Theorem 2.3. The remaining part of this section consists in proving Lemma 5.1.

Proof of Lemma 5.1. Using (1.4)-(2.1), we have

$$
\begin{aligned}
B_{n, h}^{3, \varepsilon}= & \frac{2}{(2 \pi)^{d+1}} \sum_{j_{1}, j_{2}=1}^{n} \int \mathrm{d} \omega \mathrm{d} p \mathrm{e}^{\mathrm{i} \omega\left(j_{1}-j_{2}\right) h} \int_{0}^{h} \int_{0}^{h} \mathrm{~d} u_{1} \mathrm{~d} u_{2} \mathrm{e}^{\mathrm{i} \omega\left(u_{1}-u_{2}\right)} \\
& \times \frac{a(p) \mu|p|^{2 \beta}}{|p|^{d+2(\alpha-1)}\left(\mu^{2}|p|^{4 \beta}+\omega^{2} \varepsilon^{2((1-2 \beta) s+\gamma)}\right)}\left[\sum_{r_{1}, r_{2}=1}^{N} \sum_{l_{1}, l_{2}=1}^{m_{r}} \mathrm{e}^{\mathrm{i} p \cdot\left(x_{n-j_{1}+1}^{r_{1}, l_{1}}-x_{n-j_{2}+1}^{r_{2}, l_{2}}\right)}\right. \\
& \times\left(\mathrm{e}^{\left.-\mathrm{i} p \cdot\left(y^{r_{1}, l_{1}}-y^{r_{2}, l_{2}}+h \varepsilon^{s}\left(Q_{n, j_{1}}^{r_{1}, l_{1}}-Q_{n, j_{2}}^{r_{2}, l_{2}}\right)\right) / 2\right)}-\mathrm{e}^{\left.-\mathrm{i} p \cdot\left(y^{r_{1}, l_{1}}+y^{r_{2}, l_{2}}+h \varepsilon^{s}\left(Q_{n, j_{1}}^{r_{1}, l_{1}}+Q_{n, j_{2}}^{r_{2}, l_{2}}\right)\right) / 2\right)}\right. \\
& \left.\left.-\mathrm{e}^{\left.\mathrm{i} p \cdot\left(y^{r_{1}, l_{1}}+y^{r_{2}, l_{2}}+h \varepsilon^{s}\left(Q_{n, j_{1}}^{r_{1}, l_{1}}+Q_{n, j_{2}}^{r_{2}, l_{2}}\right)\right) / 2\right)}+\mathrm{e}^{\left.\mathrm{i} p \cdot\left(y^{r_{1}, l_{1}}-y^{r_{2}, l_{2}}+h \varepsilon^{s}\left(Q_{n, j_{1}}^{r_{1}, l_{1}}-Q_{n, j_{2}}^{r_{2}, l_{2}}\right)\right) / 2\right)}\right)\right]
\end{aligned}
$$

where there is no fast phase terms anymore. Then, using the fact that $\beta<1 / 2$ or $\gamma>0$, we have

$$
\begin{aligned}
\lim _{\varepsilon \rightarrow 0} B_{n, h}^{3, \varepsilon}= & \frac{2}{(2 \pi)^{d}} \sum_{j_{1}, j_{2}=1}^{n} \int_{0}^{h} \int_{0}^{h} \mathrm{~d} u_{1} \mathrm{~d} u_{2} \delta\left(u_{1}-u_{2}+\left(j_{1}-j_{2}\right) h\right) \int \mathrm{d} p \frac{a(0)}{\mu|p|^{d+\theta}}\left[\sum_{r_{1}, r_{2}=1}^{N} \sum_{l_{1}, l_{2}=1}^{m_{r}} \mathrm{e}^{\mathrm{i} p \cdot\left(x_{n-j_{1}+1}^{r_{1}, l_{1}}-x_{n-j_{2}+1}^{r_{2}, l_{2}}\right)}\right. \\
& \left.\times\left(\mathrm{e}^{-\mathrm{i} p \cdot\left(y^{r_{1}, l_{1}}-y^{r_{2}, l_{2}}\right) / 2}-\mathrm{e}^{-\mathrm{i} p \cdot\left(y^{r_{1}, l_{1}}+y^{r_{2}, l_{2}}\right) / 2}-\mathrm{e}^{\mathrm{i} p \cdot\left(y^{r_{1}, l_{1}}+y^{r_{2}, l_{2}}\right) / 2}+\mathrm{e}^{\mathrm{i} p \cdot\left(y^{r_{1}, l_{1}}-y^{r_{2}, l_{2}}\right) / 2}\right)\right]
\end{aligned}
$$

Moreover, the integral $\int_{0}^{h} \int_{0}^{h} \mathrm{~d} u_{1} \mathrm{~d} u_{2} \delta\left(u_{1}-u_{2}+\left(j_{1}-j_{2}\right) h\right)$ is not identically zero if and only if $j_{1}=j_{2}$. Indeed, if $\left|j_{1}-j_{2}\right|>0$, the variable $u_{1}$ should belong to both $(0, h)$ and $(m h,(m+1) h)$ for some $m \in \mathbb{Z} \backslash\{0\}$, which is not possible. That concludes the proof of Lemma 5.1 .

\section{Proof of Theorem 2.4}

The proof of this theorem is a simple adaptation of the proof of Theorem 2.2. All steps are similar, we just need to show the convergence of the first and second order moments. Following the proof of Lemma 4.2, we obtain the following result.

Lemma 6.1. We have

$$
\lim _{\varepsilon \rightarrow 0} B_{n, h}^{1, \varepsilon}=-2 h \sum_{j=1}^{n} \psi\left(y+h Q_{n, j}\right), \quad \text { and } \quad \lim _{\varepsilon \rightarrow 0} B_{n, h}^{2, \varepsilon}=-2 h\left(\sum_{j=1}^{n} \psi\left(y^{1}+h Q_{n, j}^{1}\right)+\sum_{j=1}^{n} \psi\left(y^{2}+h Q_{n, j}^{2}\right)\right),
$$

where $\psi$ is defined by (2.22), $B_{n, h}^{1, \varepsilon}$ and $B_{n, h}^{2, \varepsilon}$ are respectively defined by (4.3) and (4.4), and $Q_{n, j}$ by (4.5).

Then, according to (4.2) we obtain

$$
\begin{aligned}
\lim _{\varepsilon \rightarrow 0} \mathbb{E}\left[\left\langle\widehat{W}_{n, h}^{\varepsilon}, \widehat{\phi}\right\rangle_{L^{2}}\right] & =\frac{1}{(2 \pi)^{n d}} \int \mathrm{d} Q \mathrm{~d} X \mathrm{~d} q \mathrm{~d} y \mathrm{e}^{-\mathrm{i} \sum_{j=1}^{n}\left(q_{j-1}-q_{j}\right) \cdot x_{j}} \mathrm{e}^{h \sum_{j=1}^{n} \psi\left(y+h Q_{n, j}\right)} \widehat{W}_{0}\left(q_{n}, y+h \sum_{l=1}^{n} q_{l-1}\right) \overline{\widehat{\phi}(q, y)} \\
& =\int \mathrm{d} Q \mathrm{~d} q \mathrm{~d} y \prod_{j=1}^{n} \delta\left(q_{j-1}-q_{j}\right) \mathrm{e}^{h \sum_{j=1}^{n} \psi(y+(n-j+1) h q)} \widehat{W}_{0}(q, y+n h q) \overline{\widehat{\phi}(q, y)} \\
& =\int \mathrm{d} q \mathrm{~d} y \mathrm{e}^{\psi_{n, h, \theta}(y, q)} \widehat{W}_{0}(q, y+n h q) \overline{\widehat{\phi}(q, y)}
\end{aligned}
$$


so that $\lim _{\varepsilon \rightarrow 0} \mathbb{E}\left[\left\langle W_{n, h}^{\varepsilon}, \phi\right\rangle_{L^{2}\left(\mathbb{R}^{d}\right)}\right]=\left\langle W_{n, h}, \phi\right\rangle_{L^{2}\left(\mathbb{R}^{d}\right)}$. Moreover,

$$
\begin{aligned}
\lim _{\varepsilon \rightarrow 0} \mathbb{E}\left[\left\langle\widehat{W}_{n, h}^{\varepsilon}, \widehat{\phi}\right\rangle_{L^{2}}\left\langle\widehat{W}_{n, h}^{\varepsilon}, \widehat{\phi}\right\rangle_{L^{2}}\right]= & \frac{1}{(2 \pi)^{2 n d}} \int \mathrm{d} Q^{1} \mathrm{~d} X^{1} \mathrm{~d} Q^{2} \mathrm{~d} X^{2} \mathrm{~d} q_{1} \mathrm{~d} q_{2} \mathrm{~d} y_{1} \mathrm{~d} y_{2} \overline{\widehat{\phi}\left(q_{1}, y_{1}\right) \widehat{\phi}\left(q_{2}, y_{2}\right)} \mathrm{e}^{-\mathrm{i} \sum_{j=1}^{n}\left(q_{j-1}^{1}-q_{j}^{1}\right) \cdot x_{j}^{1}} \\
& \times \mathrm{e}^{-\mathrm{i} \sum_{j=1}^{n}\left(q_{j-1}^{2}-q_{j}^{2}\right) \cdot x_{j}^{2}} \mathrm{e}^{h\left(\sum_{j=1}^{n} \psi\left(y^{1}+h Q_{n, j}^{1}\right)+\sum_{j=1}^{n} \psi\left(y^{2}+h Q_{n, j}^{2}\right)\right)} \\
& \times \widehat{W}_{0}\left(q_{n}^{1}, y^{1}+h \sum_{l=1}^{n} q_{l-1}^{1}\right) \widehat{W}_{0}\left(q_{n}^{2}, y^{2}+h \sum_{l=1}^{n} q_{l-1}^{2}\right) \\
= & \int \mathrm{d} Q^{1} \mathrm{~d} Q^{2} \mathrm{~d} q_{1} \mathrm{~d} q_{2} \mathrm{~d} y_{1} \mathrm{~d} y_{2} \overline{\widehat{\phi}\left(q_{1}, y_{1}\right) \widehat{\phi}\left(q_{2}, y_{2}\right)} \prod_{j=1}^{n} \delta\left(q_{j-1}^{1}-q_{j}^{1}\right) \delta\left(q_{j-1}^{2}-q_{j}^{2}\right) \\
& \times \mathrm{e}^{h\left(\sum_{j=1}^{n} \psi\left(y^{1}+(n-j+1) h q^{1}\right)+\sum_{j=1}^{n} \psi\left(y^{2}+(n-j+1) h q^{2}\right)\right)} \\
& \times \widehat{W}_{0}\left(q_{n}^{1}, y^{1}+n h q^{1}\right) \widehat{W}_{0}\left(q_{n}^{2}, y^{2}+n h q^{2}\right) \\
= & \int \mathrm{d} q_{1} \mathrm{~d} q_{2} \mathrm{~d} y_{1} \mathrm{~d} y_{2} \mathrm{e}^{\psi_{n, h, \theta}\left(y^{1}, q^{1}\right)+\psi_{n, h, \theta}\left(y^{2}, q^{2}\right)} \\
& \times \widehat{W}_{0}\left(q^{1}, y^{1}+n h q^{1}\right) \widehat{W}_{0}\left(q^{2}, y^{2}+n h q^{2}\right) \overline{\widehat{\phi}\left(q_{1}, y_{1}\right) \widehat{\phi}\left(q_{2}, y_{2}\right)}
\end{aligned}
$$

so that $\lim _{\varepsilon \rightarrow 0} \mathbb{E}\left[\left\langle W_{n, h}^{\varepsilon}, \phi\right\rangle_{L^{2}\left(\mathbb{R}^{2 d}\right)}^{2}\right]=\langle W(n h), \phi\rangle_{L^{2}\left(\mathbb{R}^{2 d}\right)}^{2}$. This concludes the proof of Theorem 2.4.

Acknowledgements. The authors would like to thank L. Ryzhik for stimulating discussions. This work was supported by the AFOSR FA-9550-10-1-0194 grant.

\section{REFERENCES}

[1] G. Bal, T. Komorowski and L. Ryzhik, Kinetic limits for waves in a random medium. Kinet. Relat. Models 3 (2010) 529-644.

[2] G. Bal, T. Komorowski and L. Ryzhik, Asymptotics of the phase of the solutions of the random schrödinger equation. ARMA (2011) 13-64.

[3] G. Bal and L. Ryzhik, Time splitting for wave equations in random media. ESAIM: M2AN 38 (2004) 961-988.

[4] W. Bao, S. Jin and P.A. Markowich, On Time-Splitting spectral approximations for the Schrödinger equation in the semiclassical regime. J. Comput. Phys. 175 (2002) 487-524.

[5] P. Billingsley, Convergence of Probability Measures. John Wiley and Sons, New York (1999).

[6] S. Dolan, C. Bean and R.B., The broad-band fractal nature of heterogeneity in the upper crust from petrophysical logs. Geophys. J. Int. 132 (1998) 489-507.

[7] J.-P. Fouque, J. Garnier, G. Papanicolaou and K. Sølna, Wave propagation and time reversal in randomly layered media, in vol. 56 of Stoch. Model. Appl. Probab. Springer, New York (2007).

[8] C. Gomez, Radiative transport limit for the random Schrödinger equation with long-range correlations. J. Math. Pures. Appl. 98 (2012) 295-327.

[9] C. Gomez, Wave decoherence for the random Schrödinger equation with long-range correlations. To appear in $C M P$ (2012).

[10] A.A. Gonoskov and I.A. Gonoskov, Suppression of reflection from the grid boundary in solving the time-dependent Schroedinger equation by split-step technique with fast Fourier transform, ArXiv Physics e-prints (2006).

[11] S. Jin, P. Markowich and C. Sparber, Mathematical and computational methods for semiclassical Schrödinger equations. Acta Numer. 20 (2011) 121-209.

[12] P.-L. Lions and T. Paul, Sur les mesures de Wigner. Rev. Mat. Iberoamericana 9 (1993) 553-618.

[13] P.A. Markowich, P. Pietra and C. Pohl, Numerical approximation of quadratic observables of Schrödinger-type equations in the semi-classical limit. Numer. Math. 81 (1999) 595-630.

[14] J.M. Martin and M. Flatté, Intensity images and statistics from numerical simulation of the wave propagation in 3-d random media. Appl. Optim. 247 (1988) 2111-2126.

[15] R.I. McLachlan and G.R.W. Quispel, Splitting methods. Acta Numer. 11 (2002) 341-434.

[16] C. Sidi and F. Dalaudier, Turbulence in the stratified atmosphere: Recent theoretical developments and experimental results. Adv. Space Research 10 (1990) 25-36.

[17] G. Strang, On the construction and comparison of difference schemes. SIAM J. Numer. Anal. 5 (1968) 506-517.

[18] F. Tappert, The parabolic approximation method, Wave propagation in underwater acoustics. In vol. 70 of Lect. Notes Phys. Springer (1977) 224-287. 